\title{
A SELF-SIMILAR VISCOSITY APPROACH FOR THE RIEMANN PROBLEM IN ISENTROPIC GAS DYNAMICS AND THE STRUCTURE OF THE SOLUTIONS
}

$\mathrm{BY}$

YONG JUNG KIM

Institute for Mathematics and its Applications, University of Minnesota, Minneapolis, MN

\begin{abstract}
We study the Riemann problem for the system of conservation laws of onedimensional isentropic gas dynamics in Eulerian coordinates. We construct solutions of the Riemann problem by the method of self-similar zero-viscosity limits, where the selfsimilar viscosity only appears in the equation for the conservation of momentum. No size restrictions on the data are imposed. The structure of the solutions obtained is also analyzed.
\end{abstract}

1. Introduction. We consider the equations describing one-dimensional isentropic motions of inviscid gases,

$$
\begin{aligned}
\rho_{t}+(\rho u)_{x} & =0, \\
(\rho u)_{t}+\left(\rho u^{2}+p(\rho)\right)_{x} & =0, \quad x \in \mathbb{R}, \quad t>0,
\end{aligned}
$$

in Eulerian coordinates. The functions $\rho=\rho(x, t), u=u(x, t)$ and $p=p(\rho)$ represent density, velocity and pressure in that order. The density $\rho$ takes nonnegative values and the pressure function $p(\rho)$ is smooth and defined for $\rho \geq 0$. We assume that the pressure function $p(\rho)$ satisfies the hypothesis

$$
p^{\prime}(\rho)>0 \text { for } \rho>0 .
$$

Then (1.1) forms a strictly hyperbolic system with characteristic speeds $\lambda_{ \pm}(\rho, u)=u \pm$ $\sqrt{p^{\prime}(\rho)}$ for $\rho>0$. We do not assume strict hyperbolicity for $\rho=0$ since it does not include the usual $\gamma$-laws, $p(\rho)=k \rho^{\gamma}, \gamma \geq 1, k>0$. We see that it causes significant difficulties in the analysis when a vacuum is considered. We also adopt a hypothesis

$$
p(\rho) \rightarrow \infty \text { as } \rho \rightarrow \infty \text { and } p(\rho) \rightarrow 0 \text { as } \rho \rightarrow 0,
$$

which is natural for the pressure functions of gas dynamics.

Received November 11, 1998.

2000 Mathematics Subject Classification. Primary 76N10, 35L67, 35L65.

Key words and phrases. Conservation laws, self-similar solutions, Riemann problem, isentropic gas dynamics, zero-viscosity limits.

E-mail address: yjkim@ima.umn.edu 
We are interested in the Riemann problem: finding a weak solution for (1.1) with initial data

$$
(\rho(x, 0), u(x, 0))= \begin{cases}\left(\rho_{-}, u_{-}\right), & x<0 \\ \left(\rho_{+}, u_{+}\right), & 0<x\end{cases}
$$

with $\rho_{ \pm}>0$. Since homogeneous conservation laws and Riemann initial data are invariant under the rescaling $(x, t) \rightarrow(\alpha x, \alpha t), \alpha>0$, it is natural to expect that the solution of the Riemann problem should be a function of the scaling invariance variable $\xi=x / t$, which is called the self-similar variable of the Riemann problem. A simple computation shows that $(u, \rho)(x, t)=(u, \rho)(x / t)$ is a solution of $(1.1),(1.2)$ if $(u, \rho)(\xi)$ is a solution of the boundary value problem $(\mathcal{P})$ :

$$
\begin{gathered}
-\xi \rho^{\prime}+(\rho u)^{\prime}=0, \\
-\xi(\rho u)^{\prime}+\left(\rho u^{2}+p(\rho)\right)^{\prime}=0, \\
\rho( \pm \infty)=\rho_{ \pm} . \quad u( \pm \infty)=u_{ \pm} .
\end{gathered}
$$

where the ordinary differentiation is with respect to $\xi$.

It is well known that weak solutions are not unique and that the problem (1.3) with (1.4) should be studied by introducing admissibility criteria attempting to single out the physically admissible solution. We refer to [4], [5] for the solution of the Riemann problem for general strictly hyperbolic systems and to [2] for a discussion of the issue of admissibility for hyperbolic systems of conservation laws.

In this article we study the solution of the Riemann problem $(\mathcal{P})$ as $\varepsilon \rightarrow 0$ as the limit of the solutions of the perturbed problem $\left(\mathcal{P}_{\varepsilon}\right)$ :

$$
\begin{gathered}
-\xi \rho^{\prime}+(\rho u)^{\prime}=0, \\
-\xi(\rho u)^{\prime}+\left(\rho u^{2}+p(\rho)\right)^{\prime}=\varepsilon u^{\prime \prime}, \\
\rho( \pm \infty)=\rho_{ \pm} . \quad u( \pm \infty)=u_{ \pm} .
\end{gathered}
$$

The method of self-similar viscous limits is studied in [1], [3], [10], and the approximation of $(\mathcal{P})$ with full viscosity matrices is studied in [8]. Here, we are interested in the effect of singular diffusion matrices; in mechanical models, viscosity appears in the equation of balance of momentum, but not in the equation of balance of mass.

This approach is followed by Tzavaras in [9] for the system of one-dimensional isothermal elastic response in Lagrangian coordinates. Many of the ideas used here are based on this work. In both cases the interplay of hyperbolic and parabolic aspects of the problem must be analyzed. There are two differences in the present work. While in [9] the singularity lies at a fixed point $\xi=0$ (in Lagrangian coordinates), the singularity in (1.5) is located at moving points $\xi$ with $u(\xi)=\xi$ thus depending on the solution. This leads to analyzing the free boundary problem, as can be easily seen by considering the systems

$$
\begin{gathered}
(u-\xi) \rho^{\prime}+\rho u^{\prime}=0, \\
(u-\xi) \rho u^{\prime}+p(\rho)^{\prime}=0,
\end{gathered}
$$




$$
\begin{gathered}
(u-\xi) \rho^{\prime}+\rho u^{\prime}=0, \\
(u-\xi) \rho u^{\prime}+p(\rho)^{\prime}=\varepsilon u^{\prime \prime}
\end{gathered}
$$

that are equivalent to (1.3), (1.5) for smooth solutions. The second difference is associated with complications arising from the loss of strict hyperbolicity in a vacuum. A substantial amount of the analytical effort is directed to resolving the complications associated with vacuum states.

The objectives of the article are (i) to show the existence of solutions to the problem $\left(\mathcal{P}_{\varepsilon}\right)$, (ii) to solve the Riemann problem $(\mathcal{P})$ as the $\varepsilon \rightarrow 0$ limit of solutions to $\left(\mathcal{P}_{\varepsilon}\right)$ and (iii) to study the structure of the emerging limit. We begin in Section 2 with an analysis of regularity properties and a priori estimates for weak solutions $(\rho, u)$ of $\left(\mathcal{P}_{\varepsilon}\right)$ with $\rho>0$. It is shown that such solutions are smooth except for a unique singular point, induced by the singular diffusion matrix in (1.7), and located at $u(\xi)=\xi$. It turns out that at least one of $\rho, u$ is monotone, while the other has at most one critical point.

For the existence of solutions of $\left(\mathcal{P}_{\varepsilon}\right)$ we consider a one-parameter family of boundary value problems $\left(\mathcal{P}_{\varepsilon}^{\mu}\right)$ :

$$
\begin{gathered}
(u-\xi) \rho^{\prime}+\rho u^{\prime}=0, \quad-\infty<\xi<\infty, \\
(u-\xi) \rho u^{\prime}+p(\rho)^{\prime}=\varepsilon u^{\prime \prime}, \quad 0 \leq \mu \leq \\
\rho( \pm \infty)=\rho_{ \pm}^{\mu}:=\rho_{-}+\mu\left(\rho_{ \pm}-\rho_{-}\right), \\
u( \pm \infty)=u_{ \pm}^{\mu}:=u_{-}+\mu\left(u_{ \pm}-u_{-}\right),
\end{gathered}
$$

which connect the solutions of $\left(\mathcal{P}_{\varepsilon}\right)$ to a trivial solution. In Lemmas 2.4 and 2.5 we establish a priori estimates on $u$ and $\rho$ that are used in Section 3 to construct solutions of $\left(\mathcal{P}_{\varepsilon}\right)$. In Lemma 2.4 an a priori estimate

$$
0<\delta_{\varepsilon}<\rho(\xi)
$$

is missing for the case that $u$ is increasing on $\mathbb{R}$ (this is the case when a vacuum appears in the Riemann problem). We have not been able, under the sole Hypotheses (H1) and $(\mathrm{H} 2)$, to obtain the estimate (A) for general pressure laws. Nevertheless, in Section 5 the missing estimate is established for either special pressure functions or under restrictions on the Riemann data that prevent a vacuum. (We remark that throughout the article we study solutions of $\left(\mathcal{P}_{\varepsilon}\right)$ with $\rho>0$. It is however conceivable that the problem $\left(\mathcal{P}_{\varepsilon}\right)$ admits, for special pressure laws and boundary data, solutions that vanish in density. This would run counter to the well-known fact that the solutions of the Cauchy problem for the momentum-viscosity approximation of (1.1) have the property that $\rho>0$. On the other hand, the problem $\left(\mathcal{P}_{\varepsilon}\right)$ is essentially a boundary value problem, and the possibility that it has vanishing solutions in $\rho$ cannot be a priori excluded.)

In Section 3 we apply the Leray-Schauder degree theory to a construction scheme suggested by the a priori estimates of Section 2. The obtained result on existence of the viscous problem $\left(\mathcal{P}_{\varepsilon}\right)$ is stated in Theorem 3.4 .

In Section 4 we consider a family of solutions $\left(\rho_{\varepsilon}, u_{\varepsilon}\right)$ to $\left(\mathcal{P}_{\varepsilon}\right)$ and study the limit $\varepsilon \rightarrow 0$. We show that the total variation of $\left(\rho_{\varepsilon}, u_{\varepsilon}\right)$ is uniformly bounded, and hence, by virtue of Helly's theorem, along a subsequence $\rho_{\varepsilon_{n}} \rightarrow \rho, u_{\varepsilon_{n}} \rightarrow u$ for some function $\rho \geq 0$ and $u$ of bounded variation. The emerging limit $(\rho, u)$ turns out in Theorem 4.1 to be 
a solution of the Riemamn problem $(\mathcal{P})$ constructed through the method of self-similar zero-viscosity limits.

Then we study the structure of $(\rho, u)$. For the case of convex pressure laws

$$
p^{\prime \prime}(\rho) \geq 0 \text { for } \rho>0 .
$$

the structure of $(\rho, u)$ is established in Theorem 4.7. The solution consists of two waves separated by a constant state. The waves are either a rarefaction or a shock. As an application, we consider the strictly hyperbolic case in Corollary 4.8 and show that a vacuum cannot appear in this case. Note that Hypothesis (H3) includes both cases of genuine nonlinearity and linear degeneracy.

In the last section we complete the a priori estimates for the systems with convex pressure laws. First, if the system is strictly hyperbolic, i.c., there exists a constant $c>0$ such that

$$
p^{\prime}(\rho) \geq c^{2}, \quad \rho>0,
$$

then we obtain a lower bound for $\rho$ and have a complete theory:

Theorem (Strictly hyperbolic convex laws). Suppose $p(\rho)$ satisfies (H1), (H2) and (H3). If the system (1.1) is strictly hyperbolic, then the boundary value problem $(\mathcal{P})$ has a solution $(\rho, u)$, which is the limit of the solutions of the problems $\left(\mathcal{P}_{\varepsilon}\right)$ as $\varepsilon \rightarrow 0$. The function $(\rho, u)$ has the structure stated in Theorem 4.7 and does not contain a vacuum.

Second, for convex pressure laws (not necessarily strictly convex), we exhibit a sufficient condition on the data $\left(\rho_{ \pm}, u_{ \pm}\right)$that prevents the appearance of a vacuum and completes the theory. The construction of solutions of the Riemann problem (1.1) with (1.2) via self-similar viscous limits and the structure of the emerging solution of these two cases are stated in Theorem 5.3.

2. Weak solutions and regularity properties. We consider the nonlinear boundary value problem $\left(\mathcal{P}_{\varepsilon}\right)$ :

$$
\begin{gathered}
-\xi \rho^{\prime}+(\rho u)^{\prime}=0, \\
-\xi(\rho u)^{\prime}+\left(\rho u^{2}+p(\rho)\right)^{\prime}=\varepsilon u^{\prime \prime}, \\
\rho( \pm \infty)=\rho_{ \pm}, \quad u( \pm \infty)=u_{ \pm},
\end{gathered}
$$

with fixed boundary data $\rho_{ \pm}>0, u_{ \pm}$and $0<\varepsilon<1$. In this section we study the regularity for solutions of this problem and establish a priori estimates which are used to show the existence of solutions to the problem $\left(\mathcal{P}_{\varepsilon}\right)$.

2.1. Regularity properties. First, we give a definition of the solution of $\left(\mathcal{P}_{\varepsilon}\right)$ in the weak sense.

Definition 2.1. A pair of functions $\rho>0$ and $u$ with $p(\rho), \rho \in L_{\mathrm{loc}}^{\infty}(\mathbb{R})$ and $u \in$ $W_{1 \text { loc }}^{* 1}(\mathbb{R})$ is a solution of $\left(\mathcal{P}_{\varepsilon}\right)$ if $(\rho, u)$ satisfies

$$
\begin{gathered}
\int(\zeta-u) \rho \varphi^{\prime} d \zeta+\int \rho \varphi d \zeta=0 \\
\int\left[(\zeta-u) \rho u-p(\rho)+\varepsilon u^{\prime}\right] \varphi^{\prime} d \zeta+\int \rho u \varphi d \zeta=0
\end{gathered}
$$


for all $\varphi \in C_{c}^{1}(\mathbb{R})$, continuously differentiable functions with compact support, and the essential limits $\rho( \pm \infty)$ and $u( \pm \infty)$ exist and satisfy $(2.2)$.

It is clear that the integrals in (2.3) and (2.4) are well defined. The equations (2.3) and (2.4) imply that $\rho \in L_{\text {loc }}^{\infty}(\mathbb{R})$ and $\rho u \in L_{\text {loc }}^{1}(\mathbb{R})$ are the weak derivatives of $(\xi-u) \rho$ and $(\xi-u) \rho u-p(\rho)+\varepsilon u^{\prime}$ respectively. So $(\xi-u) \rho,(\xi-u) \rho u-p(\rho)+\varepsilon u^{\prime}$ are in $W_{1 \text { loc }}^{1}(\mathbb{R})$, and hence absolutely continuous. So $-p(\rho)+\varepsilon u^{\prime}$ is also continuous.

Lemma 2.1. Let $(\rho, u)$ be a solution of $\left(\mathcal{P}_{\varepsilon}\right)$. (i) For $a, b \in \mathbb{R}$,

$$
\begin{gathered}
{[(\xi-u(\xi)) \rho(\xi)]_{a}^{b}-\int_{a}^{b} \rho(\zeta) d \zeta=0} \\
{\left[(\xi-u(\xi)) \rho(\xi) u(\xi)-p(\rho(\xi))+\varepsilon u^{\prime}(\xi)\right]_{a}^{b}-\int_{a}^{b} \rho(\zeta) u(\zeta) d \zeta=0}
\end{gathered}
$$

(ii) $u,(\xi-u) \rho$ and $-p(\rho)+\varepsilon u^{\prime}$ are continuous on $\mathbb{R}$. If $p \in C^{n}\left(\mathbb{R}^{+}\right)$for $n \geq 0$, then $\rho$ and $u$ are $C^{n+1}$ for all $\xi$ such that $\xi \neq u(\xi)$.

Proof. We already saw that $u,(\xi-u) \rho,-p(\rho)+\varepsilon u^{\prime}$ and $(\xi-u) \rho u-p(\rho)+\varepsilon u^{\prime}$ are continuous on $\mathbb{R}$. Fix $a, b \in \mathbb{R}$ with $a<b$ and consider

$$
\psi_{n}(\xi)= \begin{cases}0, & -\infty<\xi \leq a-1 / n \\ n(\xi-a)+1, & a-1 / n \leq \xi \leq a \\ 1, & a \leq \xi \leq b \\ -n(\xi-b)+1, & b \leq \xi \leq b+1 / n \\ 0, & b+1 / n \leq \xi<+\infty\end{cases}
$$

Since $\psi_{n} \notin C_{c}^{1}(\mathbb{R})$, it cannot be directly used as a test function. However, since $\psi_{n}$ is Lipschitz continuous, it can be approximated by $C_{c}^{1}$ functions. Let the sequence $\psi_{n}^{k} \in C_{c}^{1}(\mathbb{R})$ converge to $\psi_{n}$ as $k \rightarrow \infty$. If we put $\psi_{n}^{k}$ in the place of $\varphi$ in $(2.4)$, then we get

$$
\int\left[(\zeta-u) \rho u-p(\rho)+\varepsilon u^{\prime}\right]\left(\psi_{n}^{k}\right)^{\prime} d \zeta+\int \rho u \psi_{n}^{k} d \zeta=0
$$

Taking the limit $k \rightarrow \infty$, we obtain

$$
\begin{gathered}
n \int_{a-1 / n}^{a}\left[(\zeta-u) \rho u-p(\rho)+\varepsilon u^{\prime}\right] d \zeta-n \int_{b}^{b+1 / n}\left[(\zeta-u) \rho u-p(\rho)+\varepsilon u^{\prime}\right] d \zeta \\
+\int_{a-1 / n}^{b+1 / n} \rho u \psi_{n} d \zeta=0 .
\end{gathered}
$$

Since $(\zeta-u) \rho u-p(\rho)+\varepsilon u^{\prime}$ is continuous and $\left|\rho u \psi_{n}\right| \leq|\rho u| \in L_{\text {loc }}^{1}$, the Lebesgue Differentiation Theorem and the Lebesgue Dominated Convergence Theorem imply that (2.6) holds in the limit $n \rightarrow \infty$. Similar statements show (2.5). 
Let us consider (ii). Since $(\xi-u) \rho$ is continuous, $\rho$ is continuous at $\xi$ if $\xi \neq u(\xi)$. From (2.6),

$$
\begin{aligned}
\varepsilon u^{\prime}(\xi)=\int_{a}^{\xi} \rho(\zeta) u(\zeta) d \zeta-(\xi-u(\xi)) \rho(\xi) & u(\xi)+p(\rho(\xi)) \\
& +(a-u(a)) \rho(a) u(a)-p(\rho(a))+\varepsilon u^{\prime}(a)
\end{aligned}
$$

If $p$ is $C^{0}\left(\mathbb{R}^{+}\right), u$ is $C^{1}$ at $\xi \neq u(\xi)$. From $(2.5)$,

$$
(\xi-u(\xi)) \rho(\xi)=\int_{a}^{\xi} \rho(\zeta) d \zeta+(a-u(a)) \rho(a)
$$

and $\rho$ is $C^{1}$ at those points. If $p$ is $C^{n}(\mathbb{R})$, we may consider (2.7) and (2.8) $n$ more times to get $C^{n+1}$ smoothness of $\rho$ and $u$ at $\xi \neq u(\xi)$.

Lemma 2.1 indicates that singularities may arise at $s$ when $u(s)=s$, i.e., at the fixed points of $u$. Let $s$ be a singular point. Since $\rho \in L_{\text {loc }}^{\infty}(\mathbb{R}),(2.8)$ yields

$$
\int_{s}^{\xi} \rho(\zeta) d \zeta=\rho(\xi)(\xi-u(\xi))
$$

Now we prove the uniqueness of the singularity. Note that the proof is closely related with the fact that Definition 2.1 does not accept zero density.

Lemma 2.2. The singular point of a solution $(\rho, u)$ of $\left(\mathcal{P}_{\varepsilon}\right)$ is unique.

Proof. Let $s$ be a singular point and suppose there are no singular points in the interval $(s, s+\tau)$ for some $\tau>0$ small. From (2.9), we have

$$
\xi-u(\xi)=\int_{s}^{\xi} \frac{\rho(\zeta)}{\rho(\xi)} d \zeta>0, \quad \xi \in(s, s+\tau) .
$$

So $u(\xi)<\xi$ on $(s, s+\tau)$. Let us suppose there are no singular points in the interval $(s-\tau, s)$ for some $\tau>0$ small. Then

$$
\xi-u(\xi)=\int_{s}^{\xi} \frac{\rho(\zeta)}{\rho(\xi)} d \zeta<0, \quad \xi \in(s-\tau, s) .
$$

So $\xi<u(\xi)$ on $(s-\tau, s)$. From these facts, it is impossible that the graph of $y=u(\xi)$ meets the diagonal $y=\xi$ twice. Therefore, either the singular point is unique or the set of all singular points is a closed interval.

If the set of the singular points is a closed interval $[a, b]$ with $a \neq b$, then (2.5) implies $\int_{c}^{d} \rho(\zeta) d \zeta=0$ for any $[c, d] \subset[a, b]$, and thus $\rho$ vanishes on $[a, b]$. This contradicts Definition 2.1 .

2.2. Monotonicity properties. The monotonicity of solutions plays a key role in our problem. It can be easily verified that, at a point of smoothness, the solution $(\rho, u)$ of $\left(\mathcal{P}_{\varepsilon}\right)$ satisfies

$$
\begin{gathered}
(u-\xi) \rho^{\prime}+\rho u^{\prime}=0, \\
(u-\xi) \rho u^{\prime}+p(\rho)^{\prime}=\varepsilon u^{\prime \prime} .
\end{gathered}
$$


Next we analyze the behavior of $(\rho, u)$ in a neighborhood of the singular point $\xi=s$ and $\xi= \pm \infty$. From (2.11) we obtain

$$
\begin{gathered}
\rho^{\prime}=\frac{\rho u^{\prime}}{(\xi-u)}, \\
\varepsilon u^{\prime \prime}+\frac{\left\{(\xi-u)^{2}-p^{\prime}(\rho)\right\} \rho}{\xi-u} u^{\prime}=0 .
\end{gathered}
$$

(2.13) can be written in a differential form

$$
\frac{d}{d \xi}\left[u^{\prime}(\xi) \exp \left\{\frac{1}{\varepsilon} \int^{\xi} \frac{\left\{(\zeta-u)^{2}-p^{\prime}(\rho)\right\} \rho}{\zeta-u} d \zeta\right\}\right]=0,
$$

and upon integrating (2.14) we get

$$
u^{\prime}(\xi)= \begin{cases}u^{\prime}\left(\alpha_{+}\right) \exp \left\{-\frac{1}{\varepsilon} \int_{\alpha_{+}}^{\xi} \frac{\left\{(\zeta-u)^{2}-p^{\prime}(\rho)\right\} \rho}{\zeta-u} d \zeta\right. \\ u^{\prime}\left(\alpha_{-}\right) \exp \left\{-\frac{1}{\varepsilon} \int_{\alpha_{-}}^{\xi} \frac{\left\{(\zeta-u)^{2}-p^{\prime}(\rho)\right\} \rho}{\zeta-u} d \zeta\right\}, & s<\xi, \\ \xi<s\end{cases}
$$

for any $\alpha_{ \pm}$such that $s<\alpha_{+}$and $\alpha_{-}<s$, where $s$ is the unique singular point.

Since $\exp \left\{-\frac{1}{\varepsilon} \int_{\alpha_{ \pm}}^{\xi} \frac{\left\{(\zeta-u)^{2}-p^{\prime}(\rho)\right\} \rho}{\zeta-u} d \zeta\right\}$ is positive, (2.15) implies that either $u$ is strictly monotone on $(s, \infty)$ and $(-\infty, s)$ or identically constant on the intervals. It is clear from (2.12) that $\rho$ has the same monotonicity as $u$ on $(s, \infty)$ and the opposite one on $(-\infty, s)$.

The monotonicity of the positive solution $\rho \in L^{\infty}$ implies that

$$
0<k \leq \rho(\xi) \leq K<\infty, \quad \xi \in \mathbb{R}
$$

where $k$ and $K$ depend only on $\rho_{ \pm}$and $\rho(s \pm)=\lim _{\xi \rightarrow s \pm} \rho(\xi)$. Under Hypothesis (H1), $p^{\prime}(\rho)$ is bounded by

$$
0<a_{0} \leq p^{\prime}(\rho(\xi)) \leq A_{0}, \quad \xi \in \mathbb{R},
$$

where $a_{0}$ and $A_{0}$ may depend on $k$ and $K$ of $(2.16)$.

LEMma 2.3. Let $(\rho, u)$ be a solution of $\left(\mathcal{P}_{\varepsilon}\right)$ with a unique singular point $s \in \mathbb{R}$.

(i) There exist two constants $\alpha_{-}<s, \alpha_{+}>s$, depending on $a_{0}$, and a constant $\alpha>0$, depending on $a_{0}$ and $k$, such that

$$
\begin{aligned}
& \left|u^{\prime}(\xi)\right| \leq\left|u^{\prime}\left(\alpha_{+}\right)\right|\left|\frac{\xi-s}{\alpha_{+}-s}\right|^{\frac{\alpha}{\varepsilon}}, \quad s<\xi<\alpha_{+}, \\
& \left|u^{\prime}(\xi)\right| \leq\left|u^{\prime}\left(\alpha_{-}\right)\right|\left|\frac{\xi-s}{\alpha_{-}-s}\right|^{\frac{\alpha}{\varepsilon}}, \quad \alpha_{-}<\xi<s .
\end{aligned}
$$

(ii) There exist two constants $\beta_{-}<s, \beta_{+}>s$, depending on $A_{0}$, and a constant $\beta>0$, depending on $A_{0}$ and $k$, such that

$$
\begin{array}{ll}
\left|u^{\prime}(\xi)\right| \leq\left|u^{\prime}\left(\beta_{+}\right)\right| \exp \left\{-\frac{\beta}{\varepsilon}\left(\left(\frac{\xi-s}{\beta_{+}-s}\right)^{2}-1\right)\right\}, & \beta_{+}<\xi, \\
\left|u^{\prime}(\xi)\right| \leq\left|u^{\prime}\left(\beta_{-}\right)\right| \exp \left\{-\frac{\beta}{\varepsilon}\left(\left(\frac{\xi-s}{\beta_{-}-s}\right)^{2}-1\right)\right\}, & \xi<\beta_{-} .
\end{array}
$$


(iii) $u^{\prime}(s)=0$ and, for the pressure $p \in C^{n}\left(\mathbb{R}^{+}\right), n \geq 1$, the solution $(\rho, u)$ has the regularity

$$
\rho \in C(\mathbb{R}) \cap C^{n+1}(\mathbb{R}-\{s\}) ; \quad u \in C^{1}(\mathbb{R}) \cap C^{n+1}(\mathbb{R}-\{s\}) .
$$

Proof. $u(\xi) \rightarrow u_{+}$as $\xi \rightarrow \infty$ and $u^{\prime}(s+)$ is finite from $(2.7)$. We have $u(\xi)<\xi$ on $(s, \infty)$. Thus there is a positive constant $b$ such that $-b(\xi-s)+s<u(\xi)<\xi$ on $(s, \infty)$ (see Fig. 1). Let $\alpha_{+}$be a constant such that $s<\alpha_{+}<s+\frac{\theta}{b+1}$ with $\theta=\sqrt{a_{0}}$. Then for all $\zeta \in\left(s, \alpha_{+}\right)$,

$$
\frac{\left\{(\zeta-u)^{2}-p^{\prime}(\rho)\right\} \rho}{\zeta-u} \leq\left\{(1+b)\left(\alpha_{+}-s\right)^{2}-\frac{\theta^{2}}{(1+b)}\right\} \frac{\rho}{\zeta-s} \leq-\alpha \frac{1}{\zeta-s}<0
$$

with

$$
\alpha=\frac{\left(\theta^{2}-(1+b)^{2}\left(\alpha_{+}-s\right)^{2}\right) k}{(1+b)} .
$$

Then $\alpha$ is positive and, from $(2.14)$,

$$
\left|u^{\prime}(\xi)\right| \leq\left|u^{\prime}\left(\alpha_{+}\right)\right| \exp \left\{\frac{\alpha}{\varepsilon} \int_{\alpha_{+}}^{\xi} \frac{1}{\zeta-s} d \zeta\right\}=\left|u^{\prime}\left(\alpha_{+}\right)\right|\left(\frac{\xi-s}{\alpha_{+}-s}\right)^{\frac{\alpha}{\varepsilon}}
$$

for all $\xi \in\left(s, \alpha_{+}\right)$. The second statement of (i) can be proved similarly.

Now we prove (ii). Fix $\beta_{+}>s+\max \left\{2\left(u_{+}-u_{-}\right), 2 \sqrt{2} \Theta\right\}$ with $\Theta=\sqrt{A_{0}}$. Then, for any $\zeta \in\left(\beta_{+}, \infty\right), \zeta-u(\zeta) \geq \frac{1}{2}(\zeta-s)$ and

$$
\frac{\left\{(\zeta-u)^{2}-p^{\prime}(\rho)\right\} \rho}{\zeta-u} \geq\left\{\frac{1}{2}-\frac{2 \Theta^{2}}{(\zeta-s)^{2}}\right\} \rho(\zeta-s) \geq \frac{k}{4}(\zeta-s)>0 .
$$

Set

$$
\beta=\frac{\left(\beta_{+}-s\right)^{2}}{2} \frac{k}{4}
$$

Then $\beta$ is positive and

$$
\begin{aligned}
\left|u^{\prime}(\xi)\right| & \leq\left|u^{\prime}\left(\beta_{+}\right)\right| \exp \left\{-\frac{2 \beta}{\varepsilon\left(\beta_{+}-s\right)^{2}} \int_{\beta_{+}}^{\xi}(\zeta-s) d \zeta\right\} \\
& =\left|u^{\prime}\left(\beta_{+}\right)\right| \exp \left\{-\frac{\beta}{\varepsilon}\left(\left(\frac{\xi-s}{\beta_{+}-s}\right)^{2}-1\right)\right\} .
\end{aligned}
$$

The proof of the second statement of (ii) is similar. Part (i) implies regularity for $u^{\prime}$ near the singular point $\xi=s$ and especially that $u^{\prime}(s)=0$. Since $-p(\rho)+\varepsilon u^{\prime}$ is continuous, $\rho$ is also continuous (due to (H1)). So the regularity of Lemma 2.1 is improved to (2.20).

The regularity of solution $u$ will be given by the constant $\alpha$ in Lemma 2.3. If $\alpha$ can be chosen independently from $\varepsilon, u$ can be assumed as smooth as we want by taking $\varepsilon$ small enough. Note that $\alpha$ depends on the choice of $\alpha_{+}$. For example, if we take $\alpha_{+}=s+\frac{\theta}{\sqrt{2}(b+1)}$, we get $\alpha=\frac{\theta^{2}}{2(1+b)}$. Since $u^{\prime}(s)=0$, we get $b \rightarrow 0$ as $\alpha_{+} \rightarrow s$ and we also get $\alpha \rightarrow p^{\prime}(\rho(s)) \rho(s)$. So $u$ has $C^{2}$ regularity if $p^{\prime}(\rho(s)) \rho(s)>\varepsilon$. 


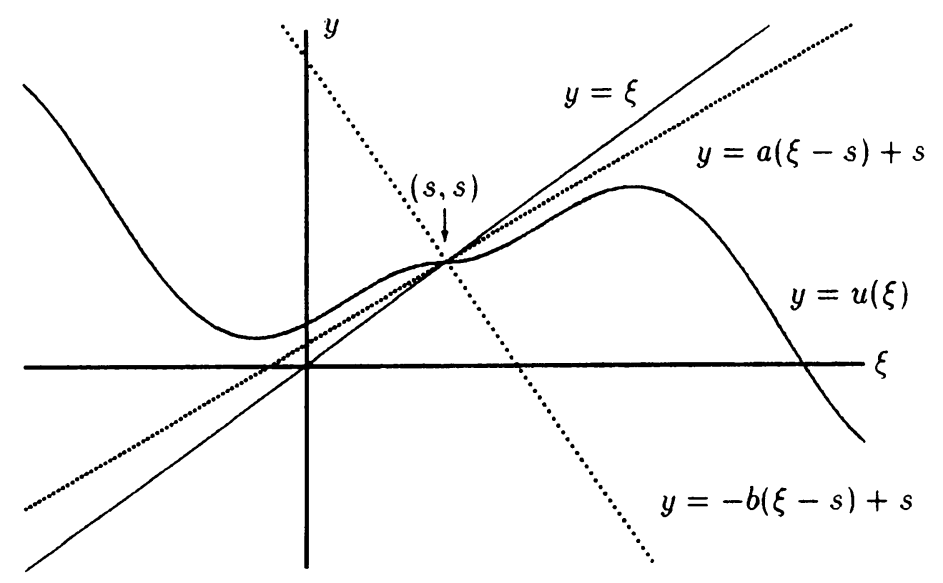

FIG. 1

2.3. A priori estimates. Throughout this section we consider a solution $(\rho, u)$ of the family of boundary value problems $\left(\mathcal{P}_{\varepsilon}^{\mu}\right)$

$$
\begin{gathered}
(u-\xi) \rho^{\prime}+\rho u^{\prime}=0, \quad-\infty<\xi<\infty, \\
(u-\xi) \rho u^{\prime}+p(\rho)^{\prime}=\varepsilon u^{\prime \prime}, \\
\rho( \pm \infty)=\rho_{ \pm}^{\mu}:=\rho_{-}+\mu\left(\rho_{ \pm}-\rho_{-}\right), \quad 0 \leq \mu \leq 1, \\
u( \pm \infty)=u_{ \pm}^{\mu}:=u_{-}+\mu\left(u_{ \pm}-u_{-}\right), \quad
\end{gathered}
$$

which connect the solutions of $\left(\mathcal{P}_{\varepsilon}\right)$ to the trivial solution associated with $\mu=0$.

Since $\rho_{ \pm}>0$, the boundary values $\rho_{ \pm}^{\mu}$ in $(2.23)$ are positive and the solutions of $\left(\mathcal{P}_{\varepsilon}^{\mu}\right)$ have the regularity derived from the previous sections. In this section we derive a priori estimates of solutions $(\rho, u)$ of $\left(\mathcal{P}_{\varepsilon}^{\mu}\right)$, which are used to establish the existence of solutions to $\left(\mathcal{P}_{\varepsilon}\right)$ in the next section. The a priori estimates are:

$$
\begin{array}{cc}
0<\delta<\rho(\xi)<M, & \xi \in(-\infty, \infty), \\
|u(\xi)|<M, & \xi \in(-\infty, \infty), \\
-b(\xi-s)+s<u(\xi)<a(\xi-s)+s, & \xi \in(s, \infty), \\
a(\xi-s)+s<u(\xi)<-b(\xi-s)+s, & \xi \in(-\infty, s),
\end{array}
$$

where positive constants $\delta$ and $M$ are independent of $\mu$, and constants $a, b$ satisfy $0<$ $a<1$ and $0<b$ and depend only on $\delta, M, \rho_{ \pm}$and $u_{ \pm}$. The point $s$ in (2.26) is the singular point of the solution, and hence it should be bounded by (2.25) and it may depend on $\mu$.

The main difference from the Lagrangian case is the estimate (2.26). It implies that the graph of the velocity $u(\xi)$ lies between two straight lines that have slopes less than 1 and pass through the point $(s, s)$ (see Fig. 1). We can easily check that $(2.26)$ is equivalent to

$$
A|\xi-s|<|u(\xi)-\xi|<B|\xi-s|, \quad \xi \neq s,
$$

with $0<A<1<B$. 
The proof of these a priori estimates strongly depends on the shape of solutions $(\rho, u)$. From the monotonicity property of the previous section, we can classify solutions into 4 categories:

$C_{1}: \rho$ is increasing on $(-\infty, \infty), u$ is decreasing on $(-\infty, s)$ and increasing on $(s, \infty)$;

$C_{2}: \rho$ is decreasing on $(-\infty, \infty), u$ is increasing on $(-\infty, s)$ and decreasing on $(s, \infty)$;

$C_{3}: \rho$ is increasing on $(-\infty, s)$ and decreasing on $(s, \infty), u$ is decreasing on $(-\infty, \infty)$;

$C_{4}: \rho$ is decreasing on $(-\infty, s)$ and increasing on $(s, \infty), u$ is increasing on $(-\infty, \infty)$. Furthermore, we may assume that the monotonicities are all strict. If not, the solution is constant on $(-\infty, s)$ or $(s, \infty)$ and the above estimates are trivial.

To establish those a priori estimates we accept the second hypothesis on the pressure function:

$$
p(\rho) \rightarrow \infty \quad \text { as } \quad \rho \rightarrow \infty \text { and } \quad p(\rho) \rightarrow 0 \quad \text { as } \quad \rho \rightarrow 0 .
$$

Lemma 2.4. Let $(\rho, u)$ be a solution of $\left(\mathcal{P}_{\varepsilon}^{\mu}\right)$. If $(\rho, u)$ belongs to the classes $C_{1}, C_{2}$ or $C_{3}$, then there exist positive constants $M$ and $\delta$ that are independent of $\mu$ and $\varepsilon$, so that $(\rho, u)$ satisfies $(2.24)$ and $(2.25)$. If $(\rho, u)$ belongs to the class of $C_{4}$, then there exists a constant $M$ that is independent of $\mu$ and $\varepsilon$ so that $(\rho, u)$ satisfies (2.24) and (2.25).

Proof. We consider (2.24) first. If $(\rho, u)$ is of class $C_{1}$ or $C_{2}$, we can take $\delta=$ $\min \left\{\rho_{-}, \rho_{+}\right\}$and $M=\max \left\{\rho_{-}, \rho_{+}\right\}$for the estimates in (2.24). We can also take $\delta=\min \left\{\rho_{-}, \rho_{+}\right\}$for the case of $C_{3}$ and $M=\max \left\{\rho_{-}, \rho_{+}\right\}$for the case of $C_{4}$. So (2.24) is completed if we prove the existence of an upper bound of $\rho$ for class $C_{3}$.

Let $\xi>s$. Then,

$$
\begin{aligned}
\rho(\xi) & =\rho_{+}^{\mu}-\int_{\xi}^{\infty} \rho^{\prime} d \zeta \leq \rho_{+}^{\mu}-\frac{1}{\xi-s} \int_{\xi}^{\infty}(\zeta-s) \rho^{\prime} d \zeta=\rho_{+}^{\mu}-\frac{1}{\xi-s} \int_{\xi}^{\infty}(\rho u)^{\prime}-s \rho^{\prime} d \zeta \\
& =\rho_{+}^{\mu}+\frac{1}{\xi-s}\left(\rho_{\xi} u_{\xi}-\rho_{+} u_{+}+s \rho_{+}-s \rho_{\xi}\right) \leq \max \left\{\rho_{-}, \rho_{+}\right\}+\frac{1}{\xi-s} \rho_{+}\left(u_{-}-u_{+}\right) .
\end{aligned}
$$

So $\rho(\xi)$ is bounded by a constant that is independent of $\mu$ and $\varepsilon$ for any fixed $\xi \neq 0$. Let $\tau \in[s+1, s+2]$ satisfy $u^{\prime}(\tau)=u(s+2)-u(s+1)>u_{+}-u_{-}$. If we integrate $(2.1)_{2}$ from $\xi>s$ to $\tau$, we get

$$
\begin{aligned}
& \rho(\xi) u^{2}(\xi)+p(\rho(\xi))-\varepsilon u^{\prime}(\xi)-\rho(\tau) u^{2}(\tau)-p(\rho(\tau))+\varepsilon u^{\prime}(\tau)=-\int_{\xi}^{\tau} \zeta(\rho u)^{\prime} d \zeta \\
& =\int_{\xi}^{\tau} \zeta(\rho(s-u))^{\prime} d \zeta-s \int_{\xi}^{\tau} \zeta \rho^{\prime} d \zeta=[\zeta \rho(s-u)]_{\xi}^{\tau}-\int_{\xi}^{\tau} \rho(s-u) d \zeta-s \int_{\xi}^{\tau}(\rho u)^{\prime} d \zeta \\
& =\tau(\rho(\tau))(s-u(\tau))-\xi(\rho(\xi))(s-u(\xi))-\int_{\xi}^{\tau} \rho(s-u) d \zeta-s \rho(\tau) u(\tau)+s \rho(\xi) u(\xi) \\
& \quad \leq \tau(\rho(\tau))(s-u(\tau))-s \rho(\tau) u(\tau)+s \rho(\xi) u(\xi) .
\end{aligned}
$$

If we take the limit $\xi \rightarrow s$, then

$$
p(\rho(s)) \leq \max _{\rho_{+}<q<\rho(s+1)}\left\{3 q \bar{u}^{2}+p(q)\right\}+\left(u_{-}-u_{+}\right)=: \mathcal{A},
$$

where $\bar{u}=\max \left\{\left|u_{-}\right|,\left|u_{+}\right|\right\} . \mathcal{A}$ is independent of $\mu$ and $\varepsilon$ and, from (H2), $p(\rho) \rightarrow \infty$ as $\rho \rightarrow \infty$. Hence $\rho(s)$ is bounded by a constant that depends only on $\rho_{ \pm}, u_{ \pm}$and the function $p$. 
Now we consider (2.25). If the solution $(\rho, u)$ is of class $C_{3}$ or $C_{4}$, we can take $M=\max \left\{u_{-}, u_{+}\right\}$for the estimation (2.25). The proof of (2.25) for solutions of class $C_{1}$ and $C_{2}$ is similar. We prove (2.25) only for the case $C_{2}$. Because of the shape of the solution we know that $|u(\xi)| \leq \max \left\{\left|u_{ \pm}\right|,|u(s)|\right\}$. So it is enough to prove that the singular point $s=u(s)$ is bounded by a constant $M$ that is independent of $\mu$ and $\varepsilon$. If $s \leq 0$, we take $M=\max \left\{\left|u_{-}\right|,\left|u_{+}\right|\right\}$. Assume that $s>0$. Since $(\xi-u)^{\prime}=1-u^{\prime}>1$ on $(s, \infty)$ and $s-u(s)=0$, there is a constant $\alpha \in(s, s+1)$ such that $\alpha-u(\alpha)=1$. Because of the monotonicity of $(\xi-u)$, we can say $(\xi-u)<1$ on $(s, \alpha)$ and $(\xi-u)>1$ on $(\alpha, \infty)$.

$$
\begin{aligned}
\rho_{+} u(\alpha) & =\rho_{+} u_{+}-\int_{\alpha}^{\infty} \rho_{+} u^{\prime} d \zeta \leq \rho_{+} u_{+}-\int_{\alpha}^{\infty} \rho u^{\prime} d \zeta \leq \rho_{+} u_{+}-\int_{\alpha}^{\infty}(\zeta-u) \rho u^{\prime} d \zeta \\
& \leq \rho_{+} u_{+}-\int_{s}^{\infty}(\zeta-u) \rho u^{\prime} d \zeta=\rho_{+} u_{+}-\int_{s}^{\infty} p(\rho)^{\prime} d \zeta+\int_{s}^{\infty} \varepsilon u^{\prime \prime} d \zeta \\
& =\rho_{+} u_{+}-p\left(\rho_{+}\right)+p(\rho(s)) \leq \rho_{+} u_{+}-p\left(\rho_{+}\right)+p\left(\rho_{-}\right) .
\end{aligned}
$$

(Note that the last inequality is from (H1).) Hence $u(\alpha) \leq u_{+}-\frac{1}{\rho_{+}}\left\{p\left(\rho_{+}\right)-p\left(\rho_{-}\right)\right\}$. Thus $u(\alpha)$ is bounded by a constant that is independent of $\varepsilon$ and $\mu$. Then

$$
\begin{aligned}
\rho_{+} u(s) & =\rho_{+} u(\alpha)-\int_{s}^{\alpha} \rho_{+} u^{\prime} d \zeta \leq \rho_{+} u(\alpha)-\int_{s}^{\alpha} \rho u^{\prime} d \zeta \\
& =\rho_{+} u(\alpha)-\int_{s}^{\alpha}(\zeta-u) \rho^{\prime} d \zeta \leq \rho_{+} u(\alpha)-\int_{s}^{\alpha} \rho^{\prime} d \zeta \\
& =\rho_{+} u(\alpha)-\rho(\alpha)+\rho(s) \leq \rho_{+} u(\alpha)-\left\{\rho_{+}-\rho_{-}\right\},
\end{aligned}
$$

and $u(s) \leq u(\alpha)+\frac{\rho_{-}}{\rho_{+}}-1$. This means that $u(s)=s$ is bounded by a constant that is independent of $\varepsilon$ and $\mu$.

The a priori estimates (2.24) and (2.25), established in Lemma 2.4, are all independent of both $\mu$ and $\varepsilon$. It is well known that if the boundary conditions $\left(\rho_{-}, u_{-}\right)$and $\left(\rho_{+}, u_{+}\right)$ are not close enough, the solution of the Riemann problem may have a vacuum state. Therefore the missing estimate, the lower bound $\delta$ of (2.24) for the case $C_{4}$, may depend on $\varepsilon$. We have been unable, under the sole Hypotheses (H1) and (H2), to establish the bound: There exists $\delta_{\varepsilon}>0$ independent of $\mu$ such that

$$
0<\delta_{\varepsilon}<\rho(\xi)
$$

for any solution of $(\rho, u)$ of Class $C_{4}$. From here on, in order to simplify the exposition, we admit (A) as an assumption and present the rest of the analysis in Sections 2 and 3 under Hypothesis (A). In Section 5 we validate (A) under additional hypotheses on the pressure law $p(\rho)$ : for strictly hyperbolic systems with convex laws (cf. Lemma 5.1), or for convex pressure laws under restrictions on $\left(\rho_{ \pm}, u_{ \pm}\right)$that exclude a vacuum (cf. Lemma 5.2).

LEMmA 2.5. Under Hypothesis (A), there exist constants $0 \leq a<1$ and $b \geq 0$, depending on $\rho_{ \pm}, u_{ \pm}$and $\delta$ and $M$ in (2.24) and (2.25), such that $(\rho, u)$ satisfies (2.26).

Proof. The proofs of $(2.26)_{1}$ and $(2.26)_{2}$ are similar and we consider $(2.26)_{1}$ only. Suppose $u$ is increasing on $(s, \infty)$. Then we can take $b=0$. Since $u(\xi)<\xi, u^{\prime}(s)=0$ and $u(\xi) \rightarrow u_{+}<\infty$ as $\xi \rightarrow \infty$, there is a constant $a \in(0,1)$ such that the line $y=a(\xi-s)+s$ 
is tangent to the graph of $u(\xi)$ and $u(\xi) \leq a(\xi-s)+s$ on $(s, \infty)$. Now we need to show that the slope $a$ is bounded above by a constant that is less than 1 and depends only on $\rho_{ \pm}, u_{ \pm}, \delta$ and $M$. Let $\xi_{1}$ be the tangential point. If the tangential point $\xi_{1}$ is far from the fixed point $s$, for example, $\xi_{1}-s>2\left(u_{+}-s\right)$, then $a<\frac{1}{2}$. Now we assume $\xi_{1}-s \leq 2\left(u_{+}-s\right)$. From (2.11) we get

$$
\left\{p^{\prime}\left(\rho\left(\xi_{1}\right)\right)-\left(u\left(\xi_{1}\right)-\xi_{1}\right)^{2}\right\} \rho^{\prime}\left(\xi_{1}\right)=\varepsilon u^{\prime \prime}\left(\xi_{1}\right) .
$$

Since $\rho^{\prime}\left(\xi_{1}\right)>0$ and $u^{\prime \prime}\left(\xi_{1}\right)<0$,

$$
\xi_{1}-u\left(\xi_{1}\right)>\sqrt{p^{\prime}\left(\rho\left(\xi_{1}\right)\right)} \geq \theta
$$

and

$$
a=\frac{u\left(\xi_{1}\right)-s}{\xi_{1}-s}<\frac{\xi_{1}-\theta-s}{\xi_{1}-s} \leq \frac{\xi_{1}-\theta}{\xi_{1}} \leq \frac{2\left(u_{+}-s\right)-\theta}{2\left(u_{+}-s\right)} .
$$

So we get $a \leq \max \left\{\frac{1}{2}, \frac{2\left(u_{+}-s\right)-\theta}{2\left(u_{+}-s\right)}\right\}$.

Now suppose $u$ is decreasing on $(s, \infty)$. Then we can take $a=0$. Since $u^{\prime}(s)=0$ and $u(\xi) \searrow u_{+}>-\infty$ as $\xi \rightarrow \infty$, there exists a positive constant $b$ such that $y=-b(\xi-s)+s$ is tangent to the graph of $y=u(\xi)$ and $u(\xi) \geq-b(\xi-s)+s$ on $(s, \infty)$. Now we need to show that the slope $-b$ is bounded below by a constant that depends only on $\rho_{ \pm}, u_{ \pm}, \delta$ and $M$. Let $\xi_{1}$ be the tangential point. Since $\left|u\left(\xi_{1}\right)-s\right|<\left|u_{+}-s\right|$, it is enough to show that $\xi_{1}-s>M_{0}$ for some constant $M_{0}$ that depends only on $\rho_{ \pm}, u_{ \pm}, \delta$ and $M$. In that case, $b \leq \frac{\left|u_{+}-s\right|}{\Lambda I_{0}}$.

Since $u^{\prime \prime}\left(\xi_{1}\right)>0$ and $\rho^{\prime}\left(\xi_{1}\right)<0,(2.28)$ yields

$$
\sqrt{a_{0}} \leq \sqrt{p^{\prime}\left(\rho\left(\xi_{1}\right)\right)} \leq\left(\xi_{1}-u\left(\xi_{1}\right)\right) .
$$

Multiplying by $\rho\left(\xi_{1}\right)$, we get

$$
\begin{gathered}
\rho\left(\xi_{1}\right) \theta \leq \rho\left(\xi_{1}\right)\left(\xi_{1}-u\left(\xi_{1}\right)\right)=\int_{s}^{\xi_{1}} \rho(\zeta) d \zeta, \\
\delta \theta \leq M \int_{s}^{\xi_{1}} d \zeta=M\left(\xi_{1}-s\right) .
\end{gathered}
$$

Hence $\xi_{1}-s \geq \frac{\delta \theta}{\Lambda I}$. The proof is complete.

This corollary is easily derived from Lemma 2.4 and the monotonicity of solutions.

Corollary 2.6. Let $\left\{\left(\rho_{\varepsilon}, u_{\varepsilon}\right)\right\}_{\varepsilon>0}$ be a family of solutions to the boundary-value problem $\left(\mathcal{P}_{\varepsilon}\right)$ corresponding to fixed data $\left(\rho_{ \pm}, u_{ \pm}\right)$. Then there exist constants $M$ and $\delta$ depending on the data such that

$$
\begin{gathered}
0<\delta \leq \rho_{\varepsilon}(\xi) \leq M, \quad\left|u_{\varepsilon}(\xi)\right| \leq M \\
T V_{(-\infty, \infty)} \rho_{\varepsilon} \leq M, \quad T V_{(-\infty, \infty)} u_{\varepsilon} \leq M,
\end{gathered}
$$

where $\delta$ may depend on $\varepsilon$ only if the solution belongs to the category $C_{4}$. 
3. Existence of solutions of $\left(\mathcal{P}_{\varepsilon}\right)$. To construct solutions of $\left(\mathcal{P}_{\varepsilon}\right)$, we apply the Leray-Schauder degree theory (Rabinowitz $[6, \mathrm{Ch} . \mathrm{V}]$ ) to a deformation of maps. Degree theory has been successful to establish connecting trajectories in problems of selfsimilar viscous limits (Dafermos [1], Slemrod and Tzavaras [8] in parabolic problems and Tzavaras [9], Slemrod [7] in hyperbolic-parabolic problems). In this work we adapt the method in [9] capturing the interplay between hyperbolic and parabolic effects in the system (2.1), but with significant modifications due to the nature of our free-boundary problem.

Let $C^{0}(\mathbb{R})$ be the Banach space of bounded continuous functions with the $C^{0}$-norm, $C^{1}(\mathbb{R})$ the Banach space of the bounded continuously differentiable functions with bounded derivatives equipped with the $C^{1}$-norm, and let

$$
X=\left\{(P, V) \in C^{0}(\mathbb{R}) \times C^{1}(\mathbb{R}):\|(P, V)\|_{X}<\infty\right\}
$$

be the Banach space equipped with the $C^{0} \times C^{1}$ norm:

$$
\|(P, V)\|_{X}=\sup _{-\infty<\xi<\infty}|P(\xi)|+\sup _{-\infty<\xi<\infty}|V(\xi)|+\sup _{-\infty<\xi<\infty}\left|V^{\prime}(\xi)\right| .
$$

For technical reasons we define two sets $Y$ (whose structure is motivated by the a priori estimates) and $\Omega$ where we will apply degree theory.

The set $Y$ consists of all $(P, V) \in X$ that are bounded by

$$
\begin{gathered}
0<\bar{\delta}<P(\xi)<\bar{M}, \quad \xi \in \mathbb{R}, \\
|V(\xi)|<\bar{M}, \quad \xi \in \mathbb{R}
\end{gathered}
$$

and that satisfy the bounds

$$
\begin{gathered}
\bar{A}<1-V^{\prime}(s)<\bar{B}, \\
\bar{A}|\xi-s|<|\xi-V(\xi)|<\bar{B}|\xi-s|, \quad \xi \neq s,
\end{gathered}
$$

for some $s \in \mathbb{R}$. Here, $\bar{\delta}, \bar{M}, \bar{A}$ and $\bar{B}$ are fixed constants that satisfy $0<\bar{\delta}<\delta, 0<M<$ $\bar{M}$ and $0<\bar{A}<A<1<B<\bar{B}$, where $M, \delta, A$ and $B$ are the constants in the estimates $(2.24),(2.25)$ and (2.27). Note that the point $s$ in (3.3) and (3.4) can be different for each $(P, V) \in Y$ and $\bar{\delta}$ is the only constant that may depend on $\varepsilon$. The geometric meaning of (3.4) is explained by Fig. 1. Note that $s$ is a fixed point of $V$ and that such fixed points are bounded by (3.2), i.e., $|s|<\bar{M}$.

The boundary value problem $\left(\mathcal{P}_{\varepsilon}^{\mu}\right)$ is transformed to a fixed point problem as follows: For a given $(P, V) \in Y$, consider the linear problem

$$
\begin{aligned}
(V-\xi) \rho^{\prime}+P u^{\prime} & =0, \\
(V-\xi) P u^{\prime}+p^{\prime}(P) \rho^{\prime} & =\varepsilon u^{\prime \prime}, \\
\rho( \pm \infty)=\rho_{ \pm}^{\mu} & :=\rho_{-}+\mu\left(\rho_{ \pm}-\rho_{-}\right), \\
u( \pm \infty)=u_{ \pm}^{\mu}: & =u_{-}+\mu\left(u_{ \pm}-u_{-}\right) .
\end{aligned}
$$

This defines an operator $\mathcal{F}:[0,1] \times Y \rightarrow X$ that carries $(\mu,(P, V)) \in[0,1] \times Y$ to the solution of (3.5) and (3.6). From the a priori estimates of Section 2, any solution $(\rho, u)$ of $\left(\mathcal{P}_{\varepsilon}^{\mu}\right)$ belongs to $Y$ and is a fixed point of the operator $\mathcal{F}(\mu, \cdot)$. 
The Leray-Schauder degree theory will be applied on a bounded open subset $\Omega$ of the Banach space $X$, which contains all possible solutions of $\left(\mathcal{P}_{\varepsilon}^{\mu}\right)$. The subset $Y \subset X$ defined by (3.1-3.4) contains all solutions of $\left(\mathcal{P}_{\varepsilon}^{\mu}\right)$ but is not bounded in the $C^{0} \times C^{1}$ norm. Next, we define the appropriate set $\Omega$.

Lemma 3.1. Let $Y \subset X$ be given by (3.1-3.4). Then, for any $K>0$, the set

$$
\Omega=\left\{(P, V) \in Y:\left|V^{\prime}(\xi)\right|<K\right\}
$$

is a bounded open subset of the Banach space $X$.

Proof. Clearly $\Omega$ is bounded. To show that it is open, we fix $\left(P_{0}, V_{0}\right) \in \Omega$ and find a small positive number $\nu$ such that $\left\|(P, V)-\left(P_{0}, V_{0}\right)\right\|_{X}<\nu$ implies $(P, V) \in \Omega$. The inequalities (3.1) and (3.2) for $(P, V)$ are easy to verify, and we just show (3.3) and (3.4).

Let $s_{0}$ be the fixed point of $V_{0}$ (i.e., $\left.V_{0}\left(s_{0}\right)=s_{0}\right)$. Since $V_{0}$ is continuous and bounded, there exist positive constants $A_{0}$ and $B_{0}$ such that

$$
\bar{A}\left|\xi-s_{0}\right|<A_{0}\left|\xi-s_{0}\right|<\left|\xi-V_{0}(\xi)\right|<B_{0}\left|\xi-s_{0}\right|<\bar{B}\left|\xi-s_{0}\right|, \quad \xi \neq s_{0} ;
$$

it also follows that $A_{0} \leq 1-V_{0}^{\prime}\left(s_{0}\right) \leq B_{0}$. Define

$$
\nu_{0}:=\min \left\{A_{0}-\bar{A}, \bar{B}-B_{0}\right\}<1
$$

and note that there exists a positive constant $\kappa<1$ such that

$$
\bar{A}+\frac{\nu_{0}}{2}<1-V_{0}^{\prime}(\xi)<\bar{B}-\frac{\nu_{0}}{2}, \quad s_{0}-\kappa<\xi<s_{0}+\kappa .
$$

Choose

$$
\nu:=\frac{1}{2} \nu_{0} \kappa \frac{\bar{A}}{\bar{B}} \leq \frac{1}{2} \nu_{0} \kappa \leq \frac{1}{2} \nu_{0}
$$

and suppose that $\left\|\left(P_{0}, V_{0}\right)-(P, V)\right\|_{X}<\nu$. Since $V$ is bounded and continuous, $V$ has a fixed point. Let $V(s)=s$. Then,

$$
\begin{gathered}
\nu \geq\left|V(s)-V_{0}(s)\right|=\left|s-V_{0}(s)\right| \geq \bar{A}\left|s-s_{0}\right|, \\
\left|s-s_{0}\right| \leq \frac{\nu}{\bar{A}},
\end{gathered}
$$

so that $\left|s-s_{0}\right| \leq \kappa \nu_{0} /(2 \bar{B})$ and, in particular, $s \in\left[s_{0}-\kappa, s_{0}+\kappa\right]$.

Since $\left|V_{0}^{\prime}(\xi)-V^{\prime}(\xi)\right|<\nu_{0} / 2,(3.7)$ implies that

$$
\bar{A}<1-V^{\prime}(\xi)<\bar{B} \quad \text { on }\left(s_{0}-\kappa, s_{0}+\kappa\right),
$$

and (3.3) is satisfied. Since $V(s)=s$, by integrating the above inequality over $(s, \xi)$, we obtain

$$
\bar{A}|\xi-s|<|\xi-V(\xi)|<\bar{B}|\xi-s|, \quad \xi \in\left(s_{0}-\kappa, s_{0}+\kappa\right), \quad \xi \neq s,
$$

so that (3.4) is satisfied on $\left(s_{0}-\kappa, s_{0}+\kappa\right)$.

On the complementary intervals $s_{0}+\kappa<\xi$ or $\xi<s_{0}-\kappa$, we have

$$
\begin{aligned}
|\xi-V(\xi)| & \leq\left|\xi-V_{0}(\xi)\right|+\nu \leq B_{0}\left|\xi-s_{0}\right|+\nu \\
& \leq \bar{B}|\xi-s|-\left(\bar{B}-B_{0}\right)|\xi-s|+B_{0}\left|s-s_{0}\right|+\nu .
\end{aligned}
$$


From the selections of the parameters

$$
\nu \leq \frac{\kappa \nu_{0}}{2 \bar{B}}, \quad\left|s-s_{0}\right| \leq \frac{\kappa \nu_{0}}{2 \bar{B}}, \quad \nu_{0} \leq \bar{B}-B_{0}, \quad \bar{B}>1
$$

and since $\xi$ takes values on the interval $|\xi-s|>\kappa\left(1-\nu_{0} /(2 \bar{B})\right)$, we get

$$
\begin{aligned}
-(\bar{B} & \left.-B_{0}\right)|\xi-s|+B_{0}\left|s-s_{0}\right|+\nu \\
& \leq-\kappa\left(\bar{B}-B_{0}\right)\left(1-\frac{\nu_{0}}{2 \bar{B}}\right)+\frac{\kappa \nu_{0} B_{0}}{2 \bar{B}}+\frac{\kappa \nu_{0}}{2 \bar{B}} \\
& \leq-\kappa\left(\bar{B}-B_{0}\right)+\frac{\kappa \nu_{0}(1+\bar{B})}{2 \bar{B}} \\
& \leq-\kappa\left(\bar{B}-B_{0}\right)\left(1-\frac{1+\bar{B}}{2 \bar{B}}\right) \leq 0 .
\end{aligned}
$$

So we have the upper bound of (3.4). The other inequality of (3.4) can be shown similarly. So $(P, V) \in \Omega$ and $\Omega$ is open.

3.1. Estimates of the operator. In this section we check that the map $\mathcal{F}$ is well defined and establish uniform estimates of $(\rho, u)=\mathcal{F}(\mu,(P, V))$ and their derivatives. The derived estimates may depend on $\rho_{ \pm}, u_{ \pm}$and $\varepsilon$ but are independent of the choice of $(\mu,(P, V)) \in[0,1] \times Y$. Consider a mapping $\mathcal{T}$ that carries $(P, V) \in Y$ to a solution $\mathcal{T}(P, V):=(\rho, u)$ of $(3.5)$ with boundary conditions

$$
\rho( \pm \infty)=\rho_{ \pm}-\rho_{-} ; \quad u( \pm \infty)=u_{ \pm}-u_{-} .
$$

It can easily be verified that $\left(\rho_{-}, u_{-}\right)+\mu \mathcal{T}(P, V)$ is a solution of (3.5) and (3.6), and hence $\mathcal{F}(\mu,(P, V))=\left(\rho_{-}, u_{-}\right)+\mu \mathcal{T}(P, V)$.

The bounds in (3.1) and Hypothesis (H1) imply the existence of positive constants $a_{0}$ and $A_{0}$ that satisfy $0<a_{0}<p^{\prime}(P(\xi))<A_{0}<\infty$ for all $\xi \in \mathbb{R}$ and depend on $\bar{\delta}$ and $\bar{M}$. From (3.5), we get

$$
\varepsilon u^{\prime \prime}+\frac{\left\{p^{\prime}(P)-(V-\xi)^{2}\right\} P}{V-\xi} u^{\prime}=0, \quad V(\xi) \neq \xi
$$

Since (3.9) has a unique singularity at the fixed point $s$ of $V, u^{\prime}$ is obtained by

$$
u^{\prime}(\xi)= \begin{cases}c_{+} \exp \left\{-\frac{1}{\varepsilon} \int_{\alpha_{+}}^{\xi} \frac{\left\{(\zeta-V)^{2}-p^{\prime}(P)\right\} P}{\zeta-V} d \zeta\right. \\
c_{-} \exp \left\{\begin{array}{ll}
\zeta & \frac{1}{\varepsilon} \int_{\alpha_{-}}^{\xi} \frac{\left\{(\zeta-V)^{2}-p^{\prime}(P)\right\} P}{\zeta-V} d \zeta
\end{array}\right\}=: c_{-} I_{-}, & \xi<s\end{cases}
$$

for any $\alpha_{-}<s<\alpha_{+}$. In turn, $\rho^{\prime}$ is obtained by $(3.5)_{1}$

$$
\rho^{\prime}(\xi)=\frac{P(\xi)}{\xi-V} u^{\prime}
$$


Lemma 3.2. Let $(P, V) \in Y$ and $V(s)=s$. Then there exist positive constants $\alpha, \alpha^{\prime}, \beta, \beta^{\prime}$ and $C_{\varepsilon}$ that depend only on $a_{0}, A_{0}, \bar{A}, \bar{B}, \bar{\delta}$ and $\bar{M}\left(C_{\varepsilon}\right.$ may depend on $\varepsilon$ ) and that satisfy

$$
\begin{gathered}
\frac{1}{C_{\varepsilon}}|\xi-S|^{\frac{\alpha^{\prime}}{\varepsilon}} \leq I_{ \pm}(\xi) \leq C_{\varepsilon}|\xi-s|^{\frac{\alpha}{\varepsilon}}, \quad|\xi-s|<1 \\
\frac{1}{C_{\varepsilon}} e^{-\frac{\beta \beta^{\prime}}{\varepsilon}(\xi-s)^{2}} \leq I_{ \pm}(\xi) \leq C_{\varepsilon} e^{-\frac{\beta}{\varepsilon}(\xi-s)^{2}}, \quad|\xi-s|>1
\end{gathered}
$$

Proof. Let $s<\xi<s+1$. Then

$$
\begin{aligned}
& \int_{\xi}^{s+1} \frac{\left\{(\zeta-V)^{2}-p^{\prime}(P)\right\} P}{\zeta-V} d \zeta=\int_{\xi}^{s+1}(\zeta-V) P d \zeta-\int_{\xi}^{s+1} \frac{p^{\prime}(P) P}{\zeta-V} d \zeta \\
& \leq \bar{M} \bar{B} \int_{0}^{1} \zeta d \zeta-\frac{a_{0} \bar{\delta}}{\bar{B}} \int_{\xi-s}^{1} \frac{1}{\zeta} d \zeta=\mathcal{A}+\alpha \log |\xi-s|
\end{aligned}
$$

where $\alpha:=\frac{a_{0} \bar{\delta}}{\bar{B}}>0$ and $\mathcal{A}:=\frac{\bar{M} \bar{B}}{2}$. Also

$$
\begin{aligned}
I_{+}(\xi) & =\exp \left\{\frac{1}{\varepsilon} \int_{\xi}^{s+1} \frac{\left\{(\zeta-V)^{2}-p^{\prime}(P)\right\} P}{\zeta-V} d \zeta\right\} \\
& \leq e^{\frac{\mathcal{A}}{\varepsilon}} e^{\frac{\alpha}{\varepsilon} \log |\xi-s|}=C_{\varepsilon}|\xi-s|^{\frac{\alpha}{\varepsilon}} .
\end{aligned}
$$

Let $s+1<\xi$. Then

$$
\begin{gathered}
-\int_{s+1}^{\xi} \frac{\left\{(\zeta-V)^{2}-p^{\prime}(P)\right\} P}{\zeta-V} d \zeta=-\int_{s+1}^{\xi}(\zeta-V) P d \zeta+\int_{s+1}^{\xi} \frac{p^{\prime}(P) P}{\zeta-V} d \zeta \\
\leq-\bar{\delta} \bar{A} \int_{1}^{\xi-s} \zeta d \zeta+\frac{A_{0} \bar{M}}{\bar{A}} \int_{1}^{\xi-s} \frac{1}{\zeta} d \zeta \leq-\beta(\xi-s)^{2}+\mathcal{A},
\end{gathered}
$$

where $\beta=\frac{\bar{\delta} \bar{A}}{2}+1$ and $\mathcal{A}$ is a positive constant that depends on $\beta$ and $\frac{A_{0} \bar{\Lambda}}{A}$. Also

$$
\begin{aligned}
I_{+}(\xi) & =\exp \left\{-\frac{1}{\varepsilon} \int_{s+1}^{\xi} \frac{\left\{(\zeta-V)^{2}-p^{\prime}(P)\right\} P}{\zeta-V} d \zeta\right\} \\
& \leq e^{\frac{\mathcal{A}}{\varepsilon}} e^{-\frac{\beta}{\varepsilon}(\xi-s)^{2}}=C_{\varepsilon} e^{-\frac{\beta}{\varepsilon}(\xi-s)^{2}} .
\end{aligned}
$$

The rest follows by similar arguments.

By Lemma 3.2, $u^{\prime}$ and $\rho^{\prime}$ are integrable on $(-\infty, \infty)$ and thus $(\rho, u)$ can be calculated by the formulas

$$
\begin{array}{r}
\rho(\xi)=\left\{\begin{array}{r}
\left(\rho_{+}-\rho_{-}\right)-c_{+} \int_{\xi}^{\infty} \frac{P(\zeta) I_{+}(\zeta)}{\zeta-V(\zeta)} d \zeta, \quad s<\xi, \\
c_{-} \int_{-\infty}^{\xi} \frac{P(\zeta) I_{-}(\zeta)}{\zeta-V(\zeta)} d \zeta, \quad \xi<s,
\end{array}\right. \\
u(\xi)=\left\{\begin{array}{r}
\left(u_{+}-u_{-}\right)-c_{+} \int_{\xi}^{\infty} I_{+}(\zeta) d \zeta, \quad s<\xi, \\
c_{-} \int_{-\infty}^{\xi} I_{-}(\zeta) d \zeta, \quad \xi<s .
\end{array}\right.
\end{array}
$$


Expressing the continuity of $(\rho, u)$ at $\xi=s$ gives

$$
\begin{aligned}
c_{+} \int_{s}^{\infty} \frac{P(\zeta) I_{+}(\zeta)}{\zeta-V(\zeta)} d \zeta+c_{-} \int_{-\infty}^{s} \frac{P(\zeta) I_{-}(\zeta)}{\zeta-V(\zeta)} d \zeta & =\rho_{+}-\rho_{-}, \\
c_{+} \int_{s}^{\infty} I_{+}(\zeta) d \zeta+c_{-} \int_{-\infty}^{s} I_{-}(\zeta) d \zeta & =u_{+}-u_{-}
\end{aligned}
$$

The determinant $\Delta$ of the linear system $(3.16)$ is

$$
\int_{s}^{\infty} \frac{P(\zeta) I_{+}(\zeta)}{\zeta-V(\zeta)} d \zeta \int_{-\infty}^{s} I_{-}(\zeta) d \zeta-\int_{-\infty}^{s} \frac{P(\zeta) I_{-}(\zeta)}{\zeta-V(\zeta)} d \zeta \int_{s}^{\infty} I_{+}(\zeta) d \zeta>0 .
$$

So there exists a unique solution $\left(c_{+}, c_{-}\right)$to $(3.16)$ and the operators $\mathcal{T}$ and $\mathcal{F}$ are well defined.

We now estimate $(\rho, u)=\mathcal{T}(P, V)$, defined by (3.14) and (3.15). Since our objective in this section is to get uniform bounds that are independent of the choice of $(P, V) \in Y$, we consider a generic constant $K_{\varepsilon}$ that may depend on $a_{0}, A_{0}, \bar{A}, \bar{B}, \bar{\delta}, \bar{M}$ and $\varepsilon$ but does not depend on $(P, V) \in Y$.

Considering the lower bounds for $I_{ \pm}$in Lemma 3.2, the determinant $\Delta$ of the linear system $(3.16)$ is bounded from below by a positive constant that depends on $a_{0}, A_{0}, \bar{A}, \bar{B}, \bar{\delta}$ and $\bar{M}$. So we get

$$
\left|c_{+}\right|+\left|c_{-}\right|<K_{\varepsilon}
$$

Now we estimate $\rho, u$ and their derivatives to see the regularity properties of the operator. Since $u^{\prime}, \rho^{\prime}$ are given by (3.10) and (3.11) and $I_{ \pm}$are bounded by (3.12) and (3.13), we have

$$
\begin{gathered}
\left|u^{\prime}(\xi)\right|<K_{\varepsilon}|\xi-s|^{\frac{\alpha}{\varepsilon}}, \quad|\xi-s|<1, \\
\left|u^{\prime}(\xi)\right|<K_{\varepsilon} e^{-\frac{\beta}{\varepsilon}(\xi-s)^{2}}, \quad|\xi-s|>1, \\
\left|\rho^{\prime}(\xi)\right|=\frac{|P(\xi)|}{|\xi-V(\xi)|}\left|u^{\prime}(\xi)\right|<K_{\varepsilon}|\xi-s|^{\frac{\alpha}{\varepsilon}-1}, \quad|\xi-s|<1, \\
\left|\rho^{\prime}(\xi)\right|=\frac{|P(\xi)|}{|\xi-V(\xi)|}\left|u^{\prime}(\xi)\right|<K_{\varepsilon} e^{-\frac{\beta}{\varepsilon}(\xi-s)^{2}}, \quad|\xi-s|>1 .
\end{gathered}
$$

We also have

$$
u^{\prime \prime}(\xi)=\frac{1}{\varepsilon} \frac{\left\{p^{\prime}(P)-(\xi-V)^{2}\right\} P}{\xi-V} c_{ \pm} I_{ \pm}, \quad \xi \neq s
$$

and

$$
\begin{aligned}
\left|u^{\prime \prime}(\xi)\right| & \leq \frac{1}{\varepsilon}\left(\frac{A_{0}}{(1-a)|\xi-s|}+(1+b)|\xi-s|\right) \bar{M} c_{ \pm} I_{ \pm} \leq K_{\varepsilon}|\xi-s|^{\frac{\alpha}{\varepsilon}-1}, \quad|\xi-s|<1, \\
\left|u^{\prime \prime}(\xi)\right| & \leq \frac{1}{\varepsilon}\left(\frac{A_{0}}{(1-a)|\xi-s|}+(1+b)|\xi-s|\right) \bar{M} c_{ \pm} I_{ \pm} \leq K_{\varepsilon} e^{-\frac{\beta}{\varepsilon}(\xi-s)^{2}}, \quad|\xi-s|>1 .
\end{aligned}
$$


From these estimates we get equicontinuity of $\rho, u$ and $u^{\prime}$ on any closed set that does not contain the singular point $s$. (3.19) and (3.20) imply

$$
\left|u^{\prime}(\xi)\right|<K_{\varepsilon}, \quad-\infty<\xi<\infty,
$$

and hence $u$ is equicontinuous. From (3.12) and (3.13), we get

$$
\begin{aligned}
& \int_{s}^{\xi} I_{+}(\zeta) d \zeta<K_{\varepsilon}(\xi-s)^{\frac{\alpha}{\varepsilon}+1}, \quad \int_{s}^{\xi} \frac{P I_{+}}{|\zeta-V|} d \zeta<K_{\varepsilon}(\xi-s)^{\frac{\alpha}{\varepsilon}}, \quad s<\xi<s+1 ; \\
& \int_{\xi}^{s} I_{-}(\zeta) d \zeta<K_{\varepsilon}(s-\xi)^{\frac{\alpha}{\epsilon}+1}, \quad \int_{\xi}^{s} \frac{P I_{-}}{|\zeta-V|} d \zeta<K_{\varepsilon}(s-\xi)^{\frac{\alpha}{\varepsilon}}, \quad s-1<\xi<s ; \\
& \int_{s+1}^{\xi} I_{+}(\zeta) d \zeta<K_{\varepsilon} e^{-\frac{\beta}{\varepsilon}(\xi-s)^{2}}, \quad \int_{s+1}^{\xi} \frac{P I_{+}}{|\zeta-V|} d \zeta<K_{\varepsilon} e^{-\frac{\beta}{\varepsilon}(\xi-s)^{2}}, \quad s+1<\xi \\
& \int_{\xi}^{s-1} I_{-}(\zeta) d \zeta<K_{\varepsilon} e^{-\frac{\beta}{\varepsilon}(\xi-s)^{2}}, \quad \int_{\xi}^{s-1} \frac{P I_{-}}{|\zeta-V|} d \zeta<K_{\varepsilon} e^{-\frac{\beta}{\varepsilon}(\xi-s)^{2}}, \quad s+1<\xi .
\end{aligned}
$$

These estimates imply the boundedness of $\rho$ and $u$ :

$$
|u(\xi)|<K_{\varepsilon}, \quad|\rho(\xi)|<K_{\varepsilon}, \quad-\infty<\xi<\infty,
$$

and establish estimates for $\rho(\xi)$ and $u(\xi)$ from (3.14) and (3.15);

$$
\begin{array}{lc}
|u(\xi)-u(s)|<K_{\varepsilon}|\xi-s|^{\frac{\alpha}{\varepsilon}+1}, & |\xi-s|<1, \\
|u(\xi)-u(s)|<K_{\varepsilon} e^{-\frac{\beta}{\varepsilon}(\xi-s)^{2}}, & |\xi-s|>1, \\
|\rho(\xi)-\rho(s)|<K_{\varepsilon}|\xi-s|^{\frac{\alpha}{\varepsilon}}, & |\xi-s|<1, \\
|\rho(\xi)-\rho(s)|<K_{\varepsilon} e^{-\frac{\beta}{\varepsilon}(\xi-s)^{2}}, & |\xi-s|>1 .
\end{array}
$$

The estimates (3.26) and (3.27) imply that the image $\mathcal{T}(Y)$ of $Y$ under the mapping $\mathcal{T}$ is bounded under the $C^{0} \times C^{1}$ norm.

3.2. Existence. If $(\rho, u)$ is a solution of $\left(\mathcal{P}_{\varepsilon}^{\mu}\right)$, then $(\rho, u)$ is a fixed point under $\mathcal{F}(\mu, \cdot)$. So $(\rho, u)$ is an image under the mapping $\mathcal{F}(\mu, \cdot)$ and the previous estimate (3.26) can be considered as an a priori estimate of solutions of $\left(\mathcal{P}_{\varepsilon}^{\mu}\right)$. Now we fix $K$ of Lemma 3.1 with $\bar{K}:=K_{\varepsilon}+1$ and consider

$$
\Omega=\left\{(P, V) \in Y:\left|V^{\prime}(\xi)\right|<\bar{K}\right\} .
$$

LEMMA 3.3. The mapping $\mathcal{T}: \bar{\Omega} \rightarrow X$ is a compact operator.

Proof. First, we show that $\mathcal{T}(\bar{\Omega})$ is precompact in $X$. Let $\left(\rho_{n}, u_{n}\right)$ be a sequence in $\mathcal{T}(\bar{\Omega})$. Since $u_{n}^{\prime}$ is uniformly bounded by $(3.26), u_{n}$ is equicontinuous. The equicontinuity of $\rho_{n}$ follows from (3.21), (3.22) and (3.30) and the one of $u_{n}^{\prime}$ from (3.19), (3.24) and (3.25).

For example, we consider $\rho_{n}$. Let $\eta>0$ be given. From (3.30) there exists $\delta_{1}>0$ such that $\left|\rho_{n}\left(\xi_{1}\right)-\rho_{n}\left(\xi_{2}\right)\right|<\eta$ for all $\xi_{1}, \xi_{2} \in I=\left[-\delta_{1}, \delta_{1}\right]$. From (3.21) and (3.22), $\rho_{n}^{\prime}$ is uniformly bounded on $I^{c}=\left(-\infty,-\delta_{1}\right) \cup\left(\delta_{1}, \infty\right)$ and there exists $\delta_{2}>0$ such that $\left|\rho_{n}\left(\xi_{1}\right)-\rho_{n}\left(\xi_{2}\right)\right|<\eta$ for all $\xi_{1}, \xi_{2} \in I^{c}$ with $\left|\xi_{1}-\xi_{2}\right|<\delta_{2}$. If we take $\delta=\min \left(\delta_{1}, \delta_{2}\right)$, we see that $\left|\rho_{n}\left(\xi_{1}\right)-\rho_{n}\left(\xi_{2}\right)\right|<2 \eta$ for $\left|\xi_{1}-\xi_{2}\right|<\delta$. So $\rho_{n}$ is equicontinuous. 
From (2.25) and (2.26), $\rho_{n}, u_{n}, u_{n}^{\prime}$ are also uniformly bounded and the Ascoli-Arzela theorem implies the existence of a subsequence, rename it $\rho_{n}, u_{n}$, that converges uniformly on every compact set. By taking a subsequence again, we can assume that the singular points $s_{n}$ converge to $s$.

Let $\rho, u$ and $u_{1}$ be the limits of $\rho_{n}, u_{n}$ and $u_{n}^{\prime}$. We can easily verify that $u^{\prime}=u_{1}$ and $(\rho, u) \in X$. Since the singular points $s_{n}$ are bounded by $\bar{M}$, we can choose $L>0$ that satisfies

$$
\begin{aligned}
\left|u_{n}^{\prime}(\xi)\right|<\eta, & & L<|\xi| ; \\
\left|u_{n}(\xi)\right|<\eta, & \left|\rho_{n}(\xi)\right|<\eta, & \xi<-L ; \\
\left|u_{n}(\xi)-\left(u_{+}-u_{-}\right)\right|<\eta, & \left|\rho_{n}(\xi)-\left(\rho_{+}-\rho_{-}\right)\right|<\eta, & L<\xi
\end{aligned}
$$

from (3.20), (3.31) and (3.32). The limit $(\rho, u)$ also satisfies these estimates. From these estimates together with the fact that $\rho_{n}, u_{n}, u_{n}^{\prime}$ converge uniformly on $[-L, L]$, we can take $N$ such that

$$
\left\|\left(\rho_{n}, u_{n}\right)-(\rho, u)\right\|_{X}=\sup _{-\infty<\xi<\infty}\left|\rho_{n}-\rho\right|+\sup _{-\infty<\xi<\infty}\left|u_{n}-u\right|+\sup _{-\infty<\xi<\infty}\left|u_{n}^{\prime}-u^{\prime}\right|<6 \eta
$$

whenever $n>N$. So $\left(\rho_{n}, u_{n}\right) \rightarrow(\rho, u)$ in $X$, and $\mathcal{T}(\bar{\Omega})$ is precompact.

Now we show that the mapping $\mathcal{T}: \bar{\Omega} \rightarrow X$ is continuous. Let $\left(P_{n}, V_{n}\right) \in \bar{\Omega}$ and $\left(P_{n}, V_{n}\right) \rightarrow(P, V)$ in $X$. Let $\left(\rho_{n}, u_{n}\right)=\mathcal{T}\left(P_{n}, V_{n}\right)$ and $(\rho, u)=\mathcal{T}(P, V)$. The sequence $\left\{\left(\rho_{n}, u_{n}\right)\right\}$ has at least one limit point $\left(\rho^{0}, u^{0}\right)$. Let $\left(\rho_{n_{k}}, u_{n_{k}}\right)$ be a subsequence converging to $\left(\rho^{0}, u^{0}\right)$. Then,

$$
\begin{array}{r}
\rho_{n_{k}}(\xi)=\left\{\begin{array}{r}
\left(\rho_{+}-\rho_{-}\right)-c_{+}^{n_{k}} \int_{\xi}^{\infty} \frac{P_{n_{k}}(\zeta) I_{+}^{n_{k}}(\zeta)}{\zeta-V_{n_{k}}(\zeta)} d \zeta, \quad s_{n_{k}}<\xi, \\
c_{-}^{n_{k}} \int_{-\infty}^{\xi} \frac{P_{n_{k}}(\zeta) I_{-}^{n_{k}}(\zeta)}{\zeta-V_{n_{k}}(\zeta)} d \zeta, \quad \xi<s_{n_{k},},
\end{array}\right. \\
u_{n_{k}}(\xi)=\left\{\begin{array}{r}
\left(u_{+}-u_{-}\right)-c_{+}^{n_{k}} \int_{\xi}^{\infty} I_{+}^{n_{k}}(\zeta) d \zeta, \quad s_{n_{k}}<\xi, \\
c_{-}^{n_{k}} \int_{-\infty}^{\xi} I_{-}^{n_{k}}(\zeta) d \zeta, \quad \xi<s_{n_{k}},
\end{array}\right.
\end{array}
$$

where $c_{+}^{n_{k}}$ and $c_{-}^{n_{k}}$ are solutions of (3.16) with $I_{ \pm}^{n_{k}}$ given by

$$
I_{ \pm}^{n_{k}}(\xi)=\exp \left\{-\frac{1}{\varepsilon} \int_{ \pm 1}^{\xi} \frac{\left\{\left(\zeta-V_{n_{k}}\right)^{2}-p^{\prime}\left(P_{n_{k}}\right)\right\} P_{n_{k}}}{\zeta-V_{n_{k}}} d \zeta\right\} .
$$

Because of the bounds in (3.12) and (3.13) we can take the limit as $k \rightarrow \infty$ inside the integrals in (3.33) and (3.34). Since $\left(\rho_{n_{k}}, u_{n_{k}}\right) \rightarrow\left(\rho^{0}, u^{0}\right)$ in $X$, we get

$$
\left(\rho^{0}, u^{0}\right)=\mathcal{T}(P, V)=(\rho, u) .
$$

This says that $\left(\rho_{n}, u_{n}\right)=\mathcal{T}\left(P_{n}, V_{n}\right)$ has exactly one limit point $(\rho, u)$, i.e., $\left(\rho_{n}, u_{n}\right) \rightarrow$ $(\rho, u)$ in $X$.

We conclude with a theorem guaranteeing existence for solutions of $\left(\mathcal{P}_{\varepsilon}\right)$ under the extra assumption (A). In Section 5 we justify (A) under additional assumptions on the pressure function $p(\rho)$ or on the Riemann data $\left(\rho_{ \pm}, u_{ \pm}\right)$. 
TheOrem 3.4. Suppose a pressure function $p(\rho)$ satisfies $(\mathrm{H} 1),(\mathrm{H} 2)$. If solutions $(\rho, u)$ of $\left(\mathcal{P}_{\varepsilon}^{\mu}\right)$ satisfy the a priori lower bound $(\mathrm{A})$, then the boundary value problem $\left(\mathcal{P}_{\varepsilon}\right)$ has a solution $(\rho(\xi), u(\xi))$ for any $\varepsilon>0$.

Proof. We define the map $\mathcal{F}:[0,1] \times \bar{\Omega} \rightarrow X$ by $\mathcal{F}(\mu, P, V)=\left(\rho_{-}, u_{-}\right)+\mu \mathcal{T}(P, V)$. If $(\rho, u)$ is a solution of $(\rho, u)=\left(\rho_{-}, u_{-}\right)+\mu \mathcal{T}(\rho, u)$ in $\Omega$, then $(\rho, u)$ is a solution of $\left(\mathcal{P}_{\varepsilon}^{\mu}\right)$ with $(\rho, u) \in \Omega$. We apply the Leray-Schauder degree theory (Rabinowitz [6, Ch. V]) to solve

$$
(\rho, u)-\mu \mathcal{T}(\rho, u)=\left(\rho_{-}, u_{-}\right), \quad \mu \in[0,1] .
$$

We have already shown that $\mathcal{T}: \bar{\Omega} \rightarrow X$ is compact. The map $\mu \mathcal{T}:[0,1] \times \bar{\Omega} \rightarrow X$ is also compact, and thus the Leray-Schauder degree of $I-\mu \mathcal{T}$ is well defined. For any solution $(\rho, u)$ of $(3.35), \frac{1}{\mu}\left\{(\rho, u)-\left(\rho_{-}, u_{-}\right)\right\} \in \mathcal{T}(\bar{\Omega})$. So $u$ satisfies $(3.26)$. Hence by Lemmas 2.4 and 2.5 and the definition of $\Omega$, any solution $(\rho, u)$ of $(3.35)$ lies in the interior of $\Omega$. Therefore,

$$
d\left(I-\mu \mathcal{T}, \Omega,\left(\rho_{-}, u_{-}\right)\right)=d\left(I, \Omega,\left(\rho_{-}, u_{-}\right)\right)=1, \quad \mu \in[0,1],
$$

and (3.35) admits at least one solution for each $\mu \in[0,1]$.

4. The structure of the solution of the Riemann problem. In this section we consider a sequence $\left(\rho_{\varepsilon}, u_{\varepsilon}\right)$ of solutions of $\left(\mathcal{P}_{\varepsilon}\right)$ obeying the estimates $(2.24),(2.25)$ and (2.26). Since the bound $M$ is independent of $\varepsilon, T V_{(-\infty, \infty)} u_{\varepsilon}<2 M$ and $T V_{(-\infty, \infty)} \rho_{\varepsilon}<$ $2 M$. On account of Helly's theorem, there exists a subsequence, which we call $\left(\rho_{\varepsilon}, u_{\varepsilon}\right)$ again, such that $\left(\rho_{\varepsilon}, u_{\varepsilon}\right)$ converges pointwise to a function $(\rho, u)$ of bounded variation as $\varepsilon \rightarrow 0$. By taking further subsequences, if necessary, we assume that $\left(\rho_{\varepsilon}, u_{\varepsilon}\right)$ belongs to one of the four categories in Section 2.3. Since the singular points $s_{\varepsilon}$ of $\left(\rho_{\varepsilon}, u_{\varepsilon}\right)$ are uniformly bounded, we may also assume that $s_{\varepsilon} \rightarrow s$ as $\varepsilon \rightarrow 0$, for some $s$. The limits $\rho$ and $u$ inherit the monotonicity properties of $\rho_{\varepsilon}$ and $u_{\varepsilon}$, but the monotonicities are no longer strict.

4.1. Solution of the Riemann problem. We construct solutions of $(\mathcal{P})$ as limits of solutions $\left(\rho_{\varepsilon}, u_{\varepsilon}\right)$ of $\left(\mathcal{P}_{\varepsilon}\right)$, and study the structure of the emerging limit. First we consider the structure under the condition of $\rho>0$. In that case, we can assume that the lower bound $\delta$ for $\rho_{\varepsilon}$ is independent of $\varepsilon$ and the constants in (2.24)--(2.26) are all independent of $\varepsilon$.

Theorem 4.1. Let $\left(\rho_{\varepsilon}, u_{\varepsilon}\right), \varepsilon>0$ be the solution of $\left(\mathcal{P}_{\varepsilon}\right)$. Then there exists a subsequence $\left(\rho_{\varepsilon_{n}}, u_{\varepsilon_{n}}\right), \varepsilon_{n} \rightarrow 0$ such that the sequence of singular points $s_{\varepsilon_{n}} \rightarrow s$ and $\left(\rho_{\varepsilon_{n}}, u_{\varepsilon_{n}}\right)$ converges pointwise to a weak solution $(\rho, u)$ of $(\mathcal{P})$. Furthermore, if $\rho>0$, then there exist constants $\beta_{-}<\alpha_{-}<s<\alpha_{+}<\beta_{+}$such that

$$
(\rho(\xi) . u(\xi))=\left\{\begin{array}{rr}
\left(\rho_{-}, u_{-}\right), & \xi<\beta_{-}, \\
(\rho(s), u(s)), & \alpha_{-}<\xi<\alpha_{+}, \\
\left(\rho_{+}, u_{+}\right), & \beta_{+}<\xi .
\end{array}\right.
$$


Proof. We omit the sub-index of $\varepsilon_{n}$ and simply use $\varepsilon$ for the subsequence. The first part of the theorem has been established already and we consider the other parts. Integrate $(2.1)_{2}$ on $\left(s_{\varepsilon}, \xi\right)$ to get

$$
\varepsilon u_{\varepsilon}^{\prime}=\rho_{\varepsilon} u_{\varepsilon}^{2}+p\left(\rho_{\varepsilon}\right)-\xi \rho_{\varepsilon} u_{\varepsilon}+\int_{s_{\varepsilon}}^{\xi} \rho_{\varepsilon} u_{\varepsilon} d \zeta-p\left(\rho_{\varepsilon}\left(s_{\varepsilon}\right)\right) .
$$

Since $\rho_{\varepsilon}, u_{\varepsilon}$ and $p\left(\rho_{\varepsilon}\right)$ are bounded by a constant $M$,

$$
\left|u_{\varepsilon}^{\prime}(\xi)\right| \leq \frac{M}{\varepsilon}(|\xi|+1), \quad-\infty<\xi<\infty .
$$

If we multiply $u_{\varepsilon}$ to $(2.1)_{2}$ and integrate it on any bounded interval $\left(a_{0}, b_{0}\right)$, then integration by parts gives

$$
\varepsilon \int_{a_{0}}^{b_{0}}\left(u_{\varepsilon}^{\prime}\right)^{2} d \zeta \leq M\left(\left|a_{0}\right|+\left|b_{0}\right|+1\right)+\varepsilon M\left(u_{\varepsilon}^{\prime}\left(b_{0}\right)-u_{\varepsilon}^{\prime}\left(a_{0}\right)\right) .
$$

From (4.3), we have $\varepsilon\left(u_{\varepsilon}^{\prime}\left(b_{0}\right)-u_{\varepsilon}^{\prime}\left(a_{0}\right)\right) \leq M\left(\left|a_{0}\right|+\left|b_{0}\right|+1\right)$. So

$$
\varepsilon \int_{a_{0}}^{b_{0}}\left(u_{\varepsilon}^{\prime}\right)^{2} d \zeta \leq M\left(\left|a_{0}\right|+\left|b_{0}\right|+1\right)
$$

Let $\varphi$ be a test function with $\operatorname{supp} \varphi \subset\left[a_{0}, b_{0}\right]$. Then,

$$
\begin{aligned}
\left|\int_{-\infty}^{\infty} \varepsilon u_{\varepsilon}^{\prime \prime} \varphi d \zeta\right| & =\left|\varepsilon \int_{-\infty}^{\infty} u_{\varepsilon}^{\prime} \varphi^{\prime} d \zeta\right| \leq \varepsilon\left(\int_{a_{0}}^{b_{0}}\left(u_{\varepsilon}^{\prime}\right)^{2} d \zeta\right)^{\frac{1}{2}}\left(\int_{a_{0}}^{b_{0}}\left(\varphi^{\prime}\right)^{2} d \zeta\right)^{\frac{1}{2}} \\
& \leq \varepsilon^{\frac{1}{2}} M\left(\left|a_{0}\right|+\left|b_{0}\right|+1\right)\left(\int_{a_{0}}^{b_{0}}\left(\varphi^{\prime}\right)^{2} d \zeta\right)^{\frac{1}{2}} .
\end{aligned}
$$

Hence

$$
\lim _{\varepsilon \rightarrow 0} \varepsilon \int_{-\infty}^{\infty} u_{\varepsilon}^{\prime \prime} \varphi d \zeta=0
$$

and

$$
\begin{aligned}
\int_{-\infty}^{\infty} & \rho u(\zeta \varphi)^{\prime} d \zeta-\int_{-\infty}^{\infty}\left(\rho u^{2}+p(\rho)\right) \varphi^{\prime} d \zeta \\
& =\lim _{\varepsilon \rightarrow 0} \int_{-\infty}^{\infty} \rho_{\varepsilon} u_{\varepsilon}(\zeta \varphi)^{\prime} d \zeta-\lim _{\varepsilon \rightarrow 0} \int_{-\infty}^{\infty}\left(\rho_{\varepsilon} u_{\varepsilon}^{2}+p\left(\rho_{\varepsilon}\right)\right) \varphi^{\prime} d \zeta \\
& =\lim _{\varepsilon \rightarrow 0} \varepsilon \int_{-\infty}^{\infty} u_{\varepsilon}^{\prime \prime} \varphi d \zeta=0,
\end{aligned}
$$

that is, $(\rho, u)$ is a weak solution of $(\mathcal{P})$.

Now we consider the structure of the limit solution under the condition $\rho>0$. In that case there exist constants $\delta>0$ and $M>0$ such that

$$
\delta<\rho(\xi)<M, \quad \xi \in \mathbb{R}
$$

for all small $\varepsilon>0$. From (H1) there exist constants $a_{0}>0$ and $A_{0}>0$ such that

$$
a_{0} \leq p^{\prime}(\rho) \leq A_{0}, \quad \xi \in \mathbb{R} .
$$

So the constants $\beta_{-}<\alpha_{-}<s<\alpha_{+}<\beta_{+}$and $\alpha, \beta$ in Lemma 2.3 are independent of $\varepsilon$. Taking the limit $\varepsilon \rightarrow 0$ in Lemma 2.3, we obtain (4.1). 
If $(\rho, u)$ is a solution of $(\mathcal{P})$, then it can easily be verified that $(\rho(x, t), u(x, t))=$ $\left(\rho\left(\frac{x}{t}\right), u\left(\frac{x}{t}\right)\right)$ is a weak solution of the Riemann problem (1.1) and (1.2). So the solution of the Riemann problem has been established through the vanishing viscosity process. Theorem 4.1 provides information on the structure of solutions that do not have a vacuum state. We conclude this section with establishing the Rankine-Hugoniot jump conditions. Since $(\rho, u)$ is of bounded variation, it has right and left limits at each $\xi \in \mathbb{R}$. Let $\mathcal{S}$ be the points of jump discontinuity of either $\rho$ or $u$.

Lemma 4.2. The solution $(\rho, u)$ satisfies the Rankine-Hugoniot jump at all $\xi \in \mathcal{S}$ :

$$
\begin{gathered}
\xi[\rho(\xi+)-\rho(\xi-)]=\rho(\xi+) u(\xi+)-\rho(\xi-) u(\xi-), \\
\xi[\rho(\xi+) u(\xi+)-\rho(\xi-) u(\xi-)]=\rho(\xi+) u^{2}(\xi+)+p(\rho(\xi+))-\rho(\xi-) u^{2}(\xi-)-p(\rho(\xi-)) .
\end{gathered}
$$

Proof. We integrate $(2.5)$ over $(\xi-\delta, \xi+\delta)$, along $\left(\rho_{\varepsilon}, u_{\varepsilon}\right)$, and take the limit as $\varepsilon \rightarrow 0$, to get

$$
(\xi+\delta-u(\xi+\delta)) \rho(\xi+\delta)-(\xi-\delta-u(\xi-\delta)) \rho(\xi-\delta)=\int_{\xi-\delta}^{\xi+\delta} \rho(\zeta) d \zeta
$$

The limit of (4.12) as $\delta \rightarrow 0$ gives

$$
\xi[\rho(\xi+)-\rho(\xi-)]=\rho(\xi+) u(\xi+)-\rho(\xi-) u(\xi-)
$$

If we integrate $(2.6)$ over $(\xi-\delta, \xi+\delta)$, along $\left(\rho_{\varepsilon}, u_{\varepsilon}\right)$, and perform the same process, then we get the other equality.

4.2. Structure of the limit solutions. In this section we study the structure of the limit $(\rho, u)$ without the strict positiveness of the density limit, i.e., $\rho \geq 0$, but with the convexity hypothesis

$$
p^{\prime \prime}(\rho) \geq 0 \text { for } \quad \rho>0
$$

Hypothesis (H3) includes both cases of genuine nonlinearity and linear degeneracy. Lemma 4.3 takes two important properties of solutions to $\left(\mathcal{P}_{\varepsilon}\right)$ under Hypothesis $(\mathrm{H} 3)$. From (2.11) we obtain

$$
\left(p^{\prime}\left(\rho_{\varepsilon}\right)-\left(u_{\varepsilon}-\xi\right)^{2}\right) \rho_{\varepsilon}^{\prime}=\varepsilon u_{\varepsilon}^{\prime \prime} .
$$

LEMmA 4.3. Let $\left(\rho_{\varepsilon}, u_{\varepsilon}\right)$ be a solution of $\left(\mathcal{P}_{\varepsilon}\right)$ and $s_{\varepsilon}$ be the singular point. Then (i) if $u_{\varepsilon}$ is increasing on $\left(s_{\varepsilon}, \infty\right)$, then $u_{\varepsilon}^{\prime} \leq 1$ on $\left(s_{\varepsilon}, \infty\right)$. (ii) If $u_{\varepsilon}$ is increasing on $\left(-\infty, s_{\varepsilon}\right)$, then $u_{\varepsilon}^{\prime} \leq 1$ on $\left(-\infty, s_{\varepsilon}\right)$. (iii) If $u_{\varepsilon}$ is decreasing on $\left(s_{\varepsilon}, \infty\right)$, then there exists exactly one $\xi \in\left(s_{\varepsilon}, \infty\right)$ such that $u_{\varepsilon}^{\prime \prime}(\xi)=0$. (iv) If $u_{\varepsilon}$ is decreasing on $\left(-\infty, s_{\varepsilon}\right)$, then there exists exactly one $\xi \in\left(-\infty, s_{\varepsilon}\right)$ such that $u_{\varepsilon}^{\prime \prime}(\xi)=0$.

Proof. Let $u_{\varepsilon}$ be increasing on $\left(s_{\varepsilon}, \infty\right)$ and suppose there exist $\xi \in\left(s_{\varepsilon}, \infty\right)$ such that $u_{\varepsilon}^{\prime}(\xi)>1$. Since $u_{\varepsilon}^{\prime}\left(s_{\varepsilon}\right)=0$ and $u_{\varepsilon}^{\prime}(\xi) \rightarrow 0$ as $\xi \rightarrow \infty$, there exist $\xi_{1}, \xi_{2} \in\left(s_{\varepsilon}, \infty\right)$ such that $u_{\varepsilon}^{\prime}\left(\xi_{1}\right)=u_{\varepsilon}^{\prime}\left(\xi_{2}\right)=1, u_{\varepsilon}^{\prime \prime}\left(\xi_{1}\right)>0, u_{\varepsilon}^{\prime \prime}\left(\xi_{2}\right)<0$ and $u_{\varepsilon}^{\prime}>1$ on $\left(\xi_{1}, \xi_{2}\right)$. In that case, 
$\left(\xi-u_{\varepsilon}\right)^{2}$ is decreasing on $\left(\xi_{1}, \xi_{2}\right)$ and

$$
\begin{aligned}
& p^{\prime}\left(\rho_{\varepsilon}\left(\xi_{1}\right)\right)-\left(u_{\varepsilon}\left(\xi_{1}\right)-\xi_{1}\right)^{2}=\varepsilon \frac{u_{\varepsilon}^{\prime \prime}\left(\xi_{1}\right)}{\rho^{\prime}\left(\xi_{1}\right)}>0, \\
& p^{\prime}\left(\rho_{\varepsilon}\left(\xi_{2}\right)\right)-\left(u_{\varepsilon}\left(\xi_{2}\right)-\xi_{2}\right)^{2}=\varepsilon \frac{u_{\varepsilon}^{\prime \prime}\left(\xi_{2}\right)}{\rho^{\prime}\left(\xi_{2}\right)}<0 .
\end{aligned}
$$

Hence

$$
p^{\prime}\left(\rho_{\varepsilon}\left(\xi_{2}\right)\right)<\left(u_{\varepsilon}\left(\xi_{2}\right)-\xi_{2}\right)^{2}<\left(u_{\varepsilon}\left(\xi_{1}\right)-\xi_{1}\right)^{2}<p^{\prime}\left(\rho_{\varepsilon}\left(\xi_{1}\right)\right) .
$$

From (H3) $p^{\prime}$ is increasing for $\rho>0$. So $\rho_{\varepsilon}\left(\xi_{2}\right)<\rho_{\varepsilon}\left(\xi_{1}\right)$, which contradicts the fact that $\rho_{\varepsilon}$ is strictly increasing on $\left(s_{\varepsilon}, \infty\right)$ when $u_{\varepsilon}$ is strictly increasing on $\left(s_{\varepsilon}, \infty\right)$. A similar argument gives (ii).

Let $u_{\varepsilon}$ be decreasing on $\left(s_{\varepsilon}, \infty\right)$. Since $u_{\varepsilon}$ is decreasing on $\left(s_{\varepsilon}, \infty\right), \rho_{\varepsilon}$ is decreasing on $\left(s_{\varepsilon}, \infty\right)$, too. Since $u_{\varepsilon}^{\prime}(\xi) \rightarrow 0$ as $\xi \rightarrow \infty$ and $u_{\varepsilon}^{\prime}\left(s_{\varepsilon}\right)=0$, there exists $\xi_{1} \in\left(s_{\varepsilon}, \infty\right)$ such that $u_{\varepsilon}^{\prime \prime}\left(\xi_{1}\right)=0$. Now suppose that there is $\xi_{2}>\xi_{1}$ such that $u_{\varepsilon}^{\prime \prime}\left(\xi_{2}\right)=0$, too. If we put $\xi_{1}$ and $\xi_{2}$ into (4.13), we get

$$
\begin{aligned}
& p^{\prime}\left(\rho_{\varepsilon}\left(\xi_{1}\right)\right)-\left(u_{\varepsilon}\left(\xi_{1}\right)-\xi_{1}\right)^{2}=0, \\
& p^{\prime}\left(\rho_{\varepsilon}\left(\xi_{2}\right)\right)-\left(u_{\varepsilon}\left(\xi_{2}\right)-\xi_{2}\right)^{2}=0 .
\end{aligned}
$$

So we have

$$
p^{\prime}\left(\rho_{\varepsilon}\left(\xi_{1}\right)\right)=\left(u_{\varepsilon}\left(\xi_{1}\right)-\xi_{1}\right)^{2}<\left(u_{\varepsilon}\left(\xi_{2}\right)-\xi_{2}\right)^{2}=p^{\prime}\left(\rho_{\varepsilon}\left(\xi_{2}\right)\right) .
$$

Since $p^{\prime}$ is increasing, $\rho_{\varepsilon}\left(\xi_{1}\right) \leq \rho_{\varepsilon}\left(\xi_{2}\right)$. But $\rho_{\varepsilon}$ is a decreasing function. So there exists exactly one point $\xi \in\left(s_{\varepsilon}, \infty\right)$ such that $u_{\varepsilon}^{\prime \prime}(\xi)=0$. Property (iv) is proved similarly.

Properties (i) and (ii) in Lemma 4.3 provide the structure of rarefaction waves and (iii) and (iv) provide the structure of shock waves. Since (2.18) implies the convergence of the double index sequence $u_{\varepsilon_{1}}\left(s_{\varepsilon_{2}}\right)$ for viscous solutions $u_{\varepsilon}$ that belong to the category of $C_{1}, C_{2}, C_{3}$, we have $u(s)=s$. For the case of $C_{4}$, the convergence is from (i) and (ii) of Lemma 4.3. So we get $u(s)=s$. In the following two lemmas we study the continuity of the limit solution.

LEMma 4.4. Let a solution $(\rho, u)$ of $(\mathcal{P})$ be a limit of viscous solutions $\left(\rho_{\varepsilon}, u_{\varepsilon}\right)$ of $\left(\mathcal{P}_{\varepsilon}\right)$ and $s$ be the limit of the singular points $s_{\varepsilon}$. Then $u(s)=s$ and $\rho$ and $u$ are continuous at $\xi=s$.

Proof. First, we suppose $\rho(s)>0$. Then, from Theorem 4.1, $\rho(\xi)$ and $u(\xi)$ are constant on a neighborhood of $\xi=s$. So $\rho$ and $u$ are continuous at $\xi=s$.

Now suppose $\rho(s)=0$. Since $\rho_{ \pm}$are positive, this is possible only when $\left(\rho_{\varepsilon}, u_{\varepsilon}\right)$ belongs to Category $C_{4}$ in Section 2.3. So $u_{\varepsilon}$ are increasing on $(-\infty, \infty)$ and $\left|u_{\varepsilon}^{\prime}(\xi)\right| \leq 1$. We already know that $\left\{u_{\varepsilon}\right\}$ is uniformly bounded. The Ascoli-Arzela theorem implies that the limit $u$ is continuous on $(-\infty, \infty)$. From the Rankine-Hugoniot jump condition at $\xi=s$ we have $p(\rho(s+))=p(\rho(s-))$. Then (H1) implies $\rho(s+)=\rho(s-)$.

Lemma 4.4 implies that the limit of viscous solutions is continuous at a singular point $s=u(s)$ in both cases of shock or rarefaction waves. Now we consider the continuity of the rarefaction waves on $\mathbb{R}$. 
Lenina 4.5. Let a solution $(\rho, u)$ of $(\mathcal{P})$ be the limit of viscous solutions of $\left(\mathcal{P}_{\varepsilon}\right)$ and $s$ be a singular point, i.e., $u(s)=s$. If $u_{\varepsilon}(\xi)$ are increasing on $(s, \infty)$, then the linits $u$ and $\rho$ are continuous on $(s, x)$. If $u_{\varepsilon}(\xi)$ are increasing on $(-\infty, s)$. then the limits $u$ and $\rho$ are continuous on $(-\infty, s)$.

Proof. From Lemma 2.4. $\left|u_{\varepsilon}(\xi)\right|$ is uniformly bounded by a constant $M$ that is independent of $\varepsilon$. We also know from Lemma 4.3 that $\left|u_{\varepsilon}^{\prime}(\xi)\right|<1$. So $\left\{u_{\varepsilon}\right\}$ is uniformly bounded and equicontinuous. So, from the Ascoli-Arzela Theorem, $u$ is continuous. From $(2.11)_{1}$

$$
\left|\rho_{\varepsilon}^{\prime}\right|=\frac{\left|\rho_{\varepsilon} u_{\varepsilon}^{\prime}\right|}{\left|u_{\varepsilon}-\xi\right|}<\frac{\rho_{\varepsilon}}{\left|u_{\varepsilon}-\xi\right|} .
$$

Suppose $\rho(s)>0$. Then, $\rho$ is constant on an open set $(s, s+\delta)$ for some $\delta$ that is independent of $\varepsilon$. The constant $a$ in $(2.26)$ is independent of $\varepsilon$. So $\left|\rho_{\varepsilon}^{\prime}\right|$ is bounded above uniformly on the open interval $(s+c \infty)$ for any $c>0$. The Ascoli-Arzela theorem implies that $|\rho|$ is continuous on $(s+c, \infty)$ for any $c>0$, that is, $\rho$ is continuous on $(s . \infty)$.

Now we consider the case of $\rho(s)=0$. This case is divided into two cases.

Case 1: Suppose $\rho(\xi)>0$ on $(s . \infty)$. It is enough to prove that $\rho$ is continuous on $(s+\delta, \infty)$ for any $\delta>0$. Suppose $u(s+\delta)=s+\delta$. Then, since $u_{s}^{\prime}(\xi) \leq 1$ for all $\varepsilon$, $u(\xi)=\xi$ on $[s, s+\delta]$. From $(2.11)_{1}$, we have $\rho=0$ on $[s . s+\delta]$. So $u(s+\delta)<s+\delta$. Since $|u(\xi)-\xi|$ is increasing, we have $|u(\xi)-\xi|>s+\delta-u(s+\delta)$ on $(s+\delta . \infty)$. So. from $(4.18), p_{\varepsilon}^{\prime}$ is miformly bounded on $(s+\delta, \infty)$. Hence $\rho$ is continuous on $(s+\delta, \infty)$ by the Ascoli-Arzela theorem.

Case 2: Suppose $\rho(\xi)=0$ on $(s, s+\tau]$ and $\rho(\xi)>0$ on $(s+\tau, \infty)$ for some $\tau>0$. The proof of the continuity on $(s+\tau, \infty)$ is the same as in Case 1. We just prove the continuity at $s+\tau$. The Rankine-Hugoniot jump condition should be satisfied at $s+\tau$. If we write the condition with $\rho((s+\tau)-)=0$ we get

$$
\begin{aligned}
(s+\tau) \rho((s+\tau)+) & =\rho((s+\tau)+) u((s+\tau)+) . \\
(s+\tau) \rho((s+\tau)+) u((s+\tau)+) & =\rho((s+\tau)+) u((s+\tau)+)^{2}+p(\rho((s+\tau)+)) .
\end{aligned}
$$

So we get $u((s+\tau)+)=s+\tau$ and $p(\rho((s+\tau)+))=0$. So $\rho((s+\tau)+)=0$ and $\rho$ is continuous.

The previous lemmas provide regularity properties for the limit solution $(\rho, u)$. Let $\mathcal{S}$ be the set of points of discontinuity of $(\rho . u)$ and $\mathcal{C}$ be the set of points of continuity. If $u$ is increasing, $(\rho, u)$ is continuous from Lemma 4.5. If $u$ is decreasing, we can easily verify that there exists at most one point of discontinuity in $(-\infty, s)$ and $(s, \infty)$ from Lemma 4.3 (iii), (iv).

Now we consider the relationship between the characteristic speeds of the problem $(\mathcal{P})$ and the weak derivative of the limit solution $(\rho, u)$. Let

$$
m=\rho u, \quad U=\left(\begin{array}{c}
\rho \\
m
\end{array}\right), \quad F(U)=\left(\begin{array}{c}
m \\
\frac{m^{2}}{\rho}+p(\rho)
\end{array}\right) .
$$

We know that the eigenvalues $\lambda_{ \pm}$of $\nabla F$ are given by

$$
\lambda_{ \pm}(\rho . u)=u \pm \sqrt{p^{\prime}(\rho)}
$$


We use the notation $\lambda_{ \pm}(\xi)=\lambda_{ \pm}(\rho(\xi), u(\xi))$ and $\lambda_{ \pm}^{\varepsilon}(\xi)=\lambda_{ \pm}\left(\rho_{\varepsilon}(\xi), u_{\varepsilon}(\xi)\right)$.

Let $d \mu=\left(d \mu_{1}, d \mu_{2}\right)=\frac{d U}{d \xi}$ be the vector-valued measure that corresponds to the weak derivative of $U$, i.e., corresponding to the linear functional:

$$
\phi \rightarrow-\int \phi^{\prime}(\xi) U(\xi) d \xi, \quad \phi \in C_{c}^{1}(\mathbb{R}) .
$$

We apply the Volpert product $([11])$ of $\nabla F(U)$ and $d \mu$ to the equation $(\mathcal{P})$ to get

$$
(\widehat{\nabla F}(U)-\xi I) d \mu=0
$$

in the sense of measures, where the averaged superposition $\widehat{\nabla F}(U)$ of $U$ by $\nabla F$ is given by

$$
\widehat{\nabla F}(U)(\xi)=\int_{0}^{1} \nabla F(U(\xi-)+s(U(\xi+)-U(\xi-))) d s
$$

Let $\xi \in \mathcal{C} \cap \operatorname{supp} \mu$. Since there is at most one point of discontinuity, $\widehat{\nabla F}(U)=\nabla F(U)$ in a neighborhood of $\xi$. Suppose the determinant of $(\nabla F(U)-\xi I)$ is not zero, for example, $\operatorname{det}(\nabla F(U)-\xi I)>0$. Then there exists a neighborhood $N$ of $\xi$ such that $\operatorname{det}(\nabla F(U)-$ $\zeta I)>\delta>0$ for all $\zeta \in N$. But from (4.23), the measures $\operatorname{det}(\nabla F(U)-\zeta I) d \mu_{1.2}=0$ on $N$, which contradicts the fact that $\xi \in \operatorname{supp} \mu$. So $\xi$ is an eigenvalue of $\nabla F(U(\xi))$. We summarize these facts in a lemma:

Lemma 4.6. Let a solution $(\rho, u)$ of $(\mathcal{P})$ be a limit of viscosity solutions $\left(\rho_{\varepsilon}, u_{\varepsilon}\right)$ of $\left(\mathcal{P}_{\varepsilon}\right)$ with a singular point $s$. Let $d \mu$ be the measure of (4.22). Then we have: (i) if $u$ is increasing, then $(\rho, u)$ is continuous. If $u$ is decreasing, then there exists at most one point of discontinuity in $(-\infty, s)$ and $(s, \infty)$. (ii) If $(\rho, u)$ is continuous at $\xi \in \operatorname{supp} \mu$, $\xi=\lambda_{+}(\xi)$ on $(s, \infty)$ and $\xi=\lambda_{-}(\xi)$ on $(-\infty, s)$.

We conclude the section with a theorem which provides the structure of the limit solution $(\rho, u)$ of the viscosity solutions $\left(\rho_{\varepsilon}, u_{\varepsilon}\right)$ that obey the a priori estimates (2.24)$(2.26)$.

Theorem 4.7. Let $(\rho, u)$ be a solution of the Riemann problem $(\mathcal{P})$ through the method of self-similar zero-viscosity limits and let $s$ be the limit of singular points. (i) If $u$ is increasing on $(s, \infty)$, then $\lambda_{+}(\xi)$ is continuous on $(s, \infty)$ and

$$
\lambda_{+}(\xi)=\left\{\begin{aligned}
\lambda_{+}(s), & s<\xi<\lambda_{+}(s), \\
\xi, & \lambda_{+}(s)<\xi<\lambda_{+}\left(\rho_{+}, u_{+}\right), \\
\lambda_{+}\left(\rho_{+}, u_{+}\right), & \lambda_{+}\left(\rho_{+}, u_{+}\right)<\xi .
\end{aligned}\right.
$$

(ii) If $u$ is increasing on $(-\infty, s)$, then $\lambda_{-}(\xi)$ is continuous on $(-\infty, s)$ and

$$
\lambda_{-}(\xi)=\left\{\begin{aligned}
\lambda_{-}(s), & \lambda_{-}(s)<\xi<s, \\
\xi, & \lambda_{-}\left(\rho_{-}, u_{-}\right)<\xi<\lambda_{-}(s), \\
\lambda_{-}\left(\rho_{-}, u_{-}\right), & \xi<\lambda_{-}\left(\rho_{-}, u_{-}\right) .
\end{aligned}\right.
$$


(iii) If $u$ is decreasing on $(s, \infty)$. then $\lambda_{+}(\xi)$ has a unique discontinuity on $(s, \infty)$ and

$$
\lambda_{+}(\xi)=\left\{\begin{aligned}
\lambda_{+}(s), & s<\xi<\frac{\rho_{+} u_{+}}{\left(\rho_{+}-\rho(s)\right)}, \\
\lambda_{+}\left(\rho_{+}, u_{+}\right), & \frac{\rho_{+} u_{+}}{\left(\rho_{+}-\rho(s)\right)}<\xi .
\end{aligned}\right.
$$

(iv) If $u$ is decreasing on $(-\infty, s)$, then $u$ has a unique discontinuity on $(-\infty, s)$ and

$$
\lambda_{-}(\xi)=\left\{\begin{aligned}
\lambda_{-}(s), & \frac{\rho_{-} u_{-}}{\left(\rho_{-}-\rho(s)\right)}<\xi<s, \\
\lambda_{-}\left(\rho_{-}, u_{-}\right), & \xi<\frac{\rho_{-} u_{-}}{\left(\rho_{-}-\rho(s)\right)} .
\end{aligned}\right.
$$

Proof. We always consider the eigenvalue $\lambda_{-}$on the interval $(-\infty, s)$ and $\lambda_{+}$on the other side $(s, \infty)$. If $u$ is increasing, then $u$ and $\rho$ are continuous. So they should be constant out of $\operatorname{supp} \mu$ and $\lambda_{ \pm}$are continuous and increasing, too. With these facts we can easily check that $\operatorname{supp} \mu$ is a connected sub-interval of $(-\infty, s]$ or $[s, \infty)$. If not, $\lambda_{ \pm}$ is discontinuous. So the structure of $\lambda_{ \pm}$should follow (i) and (ii).

If $u$ is decreasing, then $\lambda_{ \pm}$are also decreasing and there exists at most one point of discontinuity on $(-\infty, s)$ and $(s, \infty)$. Since $\lambda_{ \pm}=\xi$ on supp $\mu$ and $\lambda_{ \pm}$are decreasing, $\operatorname{supp} \mu$ should be the point of discontinuity and $\lambda_{ \pm}$should be constant before and after the discontinuity. We can find the point of the discontinuity from the Rankine-Hugoniot jump condition, and $\lambda_{ \pm}$should follow (iii) and (iv).

In summary, the limit of viscosity solutions has an intermediate state which is connected to the boundary states by rarefaction waves ((i) and (ii)) or shocks ((iii) and (iv)).

COROllary 4.8. If the system (1.1) is strictly hyperbolic, i.e., there exists $c>0$ such that

$$
p^{\prime}(\rho) \geq c^{2}>0 . \quad \rho>0
$$

then the emerging limit does not have a vacuum state.

Proof. Theorem 4.7 implies that $\lambda_{+}(\xi)$ is constant on the interval $\left(s, \lambda_{+}(s)\right) \neq \varnothing$ and $\left(\lambda_{+}\left(\rho_{+}, u_{+}\right), \infty\right)$. Since $\sqrt{p^{\prime}(\rho)}$ is increasing on $(s, \infty), u(\xi)$ is also constant on those intervals. From $(1.6)_{1} \rho$ is also constant. Now we consider the interval $\left(\lambda_{+}(s), \lambda_{+}\left(\rho_{+}, u_{+}\right)\right)$. From $(1.6)_{2}$ we get $c^{2} \rho^{\prime} \leq(\xi-u) \rho$, and hence there exists a constant $C$ such that

$$
\rho^{\prime} \leq C \rho .
$$

So we have

$$
\rho(\xi) \leq \rho\left(\lambda_{+}(s)\right) e^{C\left(\xi-\lambda_{+}(s)\right)} .
$$

Suppose the solution has a vacuum state, i.e., $\rho(s)=0$. Then, since $\rho$ is constant on $\left(s, \lambda_{+}(s)\right), \rho\left(\lambda_{+}(s)\right)=0$, and hence $\rho$ is zero on $\left(\lambda_{+}(s), \lambda_{+}\left(\rho_{+}, u_{+}\right)\right)$. So $\rho(\infty)=0$, which contradicts the boundary condition $\rho(\infty)=\rho_{+}>0$.

5. Convex pressure laws. In Lemma 2.4 the a priori estimates (2.24), (2.25) are established except for the lower bound for $\rho$ of the case $C_{4}$. In this section we complete the a priori estimates in two cases under the convex pressure laws (H3). First, we consider the case of strictly hyperbolic systems. 
The equation $(2.22)_{1}$ can be written as a first-order linear equation for $\rho$ :

$$
\rho^{\prime}+\frac{u^{\prime}}{u-\xi} \rho=0, \quad \xi \neq s,
$$

where $s$ is the singular point. Then the solution is given by

$$
\rho(\xi)= \begin{cases}\rho_{+}^{\mu} e^{-\int_{\xi}^{\infty} \frac{u^{\prime}}{\zeta-u} d \zeta}, & s<\xi, \\ \rho_{-}^{\mu} e^{-\int_{-\infty}^{\xi} \frac{u^{\prime}}{u-\zeta} d \zeta}, & \xi<s,\end{cases}
$$

where $\rho_{-}^{\mu}=\rho_{-}$and $\rho_{+}^{\mu}=\rho_{-}+\mu\left(\rho_{+}-\rho_{-}\right)$are the boundary conditions of (2.23).

Lemma 5.1. Let a solution $(\rho, u)$ of $\left(\mathcal{P}_{\varepsilon}^{\mu}\right)$ belong to the class $C_{4}$. If the system (1.1) is strictly hyperbolic, i.e.,

$$
p^{\prime}(\rho) \geq c^{2}>0, \quad \rho>0,
$$

then there exists a constant $\delta>0$ that satisfies (2.24) and is independent of $\varepsilon$ and $\mu$.

Proof. Since $u$ is increasing on $\mathbb{R}, u^{\prime} \leq 1$ by Lemma 4.3 and $\xi-u(\xi)$ is also increasing. So there exists $\xi_{1}>s$ such that $0<\xi-u(\xi) \leq \frac{c}{2}$ on $\left(s, \xi_{1}\right)$ and $\frac{c}{2} \leq \xi-u(\xi)$ on $\left(\xi_{1}, \infty\right)$. Then

$$
\rho\left(\xi_{1}\right)=\rho_{+}^{\mu} e^{-\int_{\xi_{1}}^{\infty} \frac{u^{\prime}}{\zeta-u} d \zeta} \geq \rho_{+}^{\mu} e^{-\frac{2}{c}\left(u_{+}-u_{-}\right)} .
$$

Integrating $(2.28)$ on $\left(s, \xi_{1}\right)$ we get

$$
\varepsilon \int_{s}^{\xi_{1}} u^{\prime \prime}(\zeta) d \zeta=\varepsilon u^{\prime}\left(\xi_{1}\right) \leq \varepsilon
$$

and

$$
\int_{s}^{\xi_{1}}\left(p^{\prime}(\rho)-(\zeta-u)^{2}\right) \rho^{\prime} d \zeta \geq \frac{3 c^{2}}{4} \int_{s}^{\xi_{1}} \rho^{\prime} d \zeta \geq \frac{3 c^{2}}{4}\left(\rho\left(\xi_{1}\right)-\rho(s)\right)
$$

From the above estimations $\rho$ is bounded below by

$$
\rho(s) \geq \rho_{+}^{\mu} e^{-\frac{2}{c}\left(u_{+}-u_{-}\right)}-\frac{4 \varepsilon}{3 c^{2}} \geq \min \left\{\rho_{-}, \rho_{+}\right\} e^{-\frac{2}{c}\left(u_{+}-u_{-}\right)}-\frac{4 \varepsilon}{3 c^{2}} .
$$

The positive lower bound for $\rho$ is obtained for small $\varepsilon$.

We return to general convex pressure laws (H3) and consider the function

$$
g(\rho)=\frac{p(\rho)}{\rho}, \quad \rho>0 .
$$

Either the function $g: \mathbb{R}^{+} \rightarrow \mathbb{R}^{+}$is invertible or the system is strictly hyperbolic. Consider the case when $g$ has an inverse $g^{-1}$.

Lemma 5.2. Let a solution $(\rho, u)$ of $\left(\mathcal{P}_{\varepsilon}^{\mu}\right)$ belong to the class $C_{4}$. If the boundary conditions $\left(\rho_{ \pm}, u_{ \pm}\right)$satisfy

$$
u_{+}-u_{-}<\max _{m>0}\left(m \ln \left(\frac{\rho_{-}^{\mu}}{g^{-1}\left(m^{2}\right)}\right)\right)+\max _{m>0}\left(m \ln \left(\frac{\rho_{+}^{\mu}}{g^{-1}\left(m^{2}\right)}\right)\right),
$$

then there exists a constant $\delta>0$ that satisfies (2.24) and is independent of $\varepsilon$ and $\mu$. 
Proof. Let $s$ be the singular point of the solution $(\rho, u)$. Since $u$ is increasing in $\mathbb{R}$, we have $u_{-}<u(s)<u_{+}$. If $(5.9)$ holds,

$$
u_{+}-u(s)<\max _{m>0}\left(m \ln \left(\frac{\rho_{+}^{\mu}}{g^{-1}\left(m^{2}\right)}\right)\right)
$$

or

$$
u(s)-u_{-}<\max _{m>0}\left(m \ln \left(\frac{\rho_{-}^{\mu}}{g^{-1}\left(m^{2}\right)}\right)\right) .
$$

We assume that (5.10) holds. Then there exists $m>0$ such that $u_{+}-u(s)<$ $m \ln \left(\frac{\rho_{+}^{\mu}}{g^{-1}\left(m^{2}\right)}\right)$, or, equivalently,

$$
g\left(\rho_{+}^{\mu} e^{-\frac{\left(u_{+}-u(s)\right)}{m}}\right)-m^{2}>0 .
$$

Since $\xi-u(\xi)$ is increasing, there exists $\xi_{1}>s$ such that $0<\xi-u(\xi) \leq m$ on $\left(s, \xi_{1}\right)$ and $m \leq \xi-u(\xi)$ on $\left(\xi_{1}, \infty\right)$. Then

$$
\rho\left(\xi_{1}\right)=\rho_{+}^{\mu} e^{-\int_{\xi_{1}}^{\infty} \frac{u^{\prime}}{\zeta-u} d \zeta} \geq \rho_{+}^{\mu} e^{-\frac{\left(u_{+}-u(s)\right)}{m}} .
$$

We can easily check that $g(\rho)$ is increasing for $\rho>0$ and $g\left(\rho\left(\xi_{1}\right)\right)-m^{2}>0$. Integrating $(2.28)$ on $\left(s, \xi_{1}\right)$ we get

$$
\varepsilon \int_{s}^{\xi_{1}} u^{\prime \prime}(\zeta) d \zeta=\varepsilon u^{\prime}\left(\xi_{1}\right) \leq \varepsilon
$$

and

$$
\int_{s}^{\xi_{1}}\left(p^{\prime}(\rho)-(\zeta-u)^{2}\right) \rho^{\prime} d \xi \geq\left(\frac{p\left(\rho\left(\xi_{1}\right)\right)-p(\rho(s))}{\rho\left(\xi_{1}\right)-\rho(s)}-m^{2}\right)\left(\rho\left(\xi_{1}\right)-\rho(s)\right) .
$$

The convexity Hypothesis (H3) implies

$$
\frac{p\left(\rho\left(\xi_{1}\right)\right)-p(\rho(s))}{\rho\left(\xi_{1}\right)-\rho(s)}-m^{2}>\frac{p\left(\rho\left(\xi_{1}\right)\right)}{\rho\left(\xi_{1}\right)}-m^{2}=g\left(\rho\left(\xi_{1}\right)\right)-m^{2}>0 .
$$

So the density $\rho$ is bounded below by

$$
\rho(s) \geq \min \left\{\rho_{+}, \rho_{-}\right\} e^{-\frac{1}{m}\left(u_{+}-u(s)\right)}-\frac{\varepsilon}{g\left(\xi_{1}\right)-m^{2}}>0
$$

for a sufficiently small $\varepsilon>0$. The situation is similar if (5.11) holds.

One can check that, if

$$
u_{+}-u_{-}<\max _{m>0}\left(m \ln \left(\frac{\rho_{-}}{g^{-1}\left(m^{2}\right)}\right)\right)+\max _{m>0}\left(m \ln \left(\frac{\rho_{+}}{g^{-1}\left(m^{2}\right)}\right)\right)
$$

holds and $\rho_{-} \leq \rho_{+}$, then (5.9) holds. If $\rho_{-}>\rho_{+}$and instead of using the continuation of the boundary data (2.23) one uses

$$
\begin{aligned}
& \rho( \pm \infty)=\rho_{ \pm}^{\mu}:=\rho_{+}+\mu\left(\rho_{ \pm}-\rho_{+}\right), \\
& u( \pm \infty)=u_{ \pm}^{\mu}:=u_{+}+\mu\left(u_{ \pm}-u_{+}\right),
\end{aligned}
$$


then again (5.9) holds. Thus (5.18) provides a sufficient condition for preventing a vacuum from appearing. It is known that admissible solutions of (1.1) and (1.2) do not have a vacuum state if and only if

$$
u_{+}-u_{-}<\int_{0}^{\rho_{-}} \frac{\sqrt{p^{\prime}(\rho)}}{\rho} d \rho+\int_{0}^{\rho_{+}} \frac{\sqrt{p^{\prime}(\rho)}}{\rho} d \rho .
$$

While (5.18) is a sufficient condition to avoid a vacuum, simple numerical computations show that it is not a necessary condition. In the case of $\gamma$-laws, $p(\rho)=\rho^{\gamma}$ for $\gamma>1$, the condition (5.18) corresponds to

$$
u_{+}-u_{-}<\ln \left(\frac{2}{\gamma-1}\right)\left[\left(\frac{2}{\rho_{-}(\gamma-1)}\right)^{\frac{1-\gamma}{2}}+\left(\frac{2}{\rho_{+}(\gamma-1)}\right)^{\frac{1-\gamma}{2}}\right] .
$$

Now we summarize the previous lemmas and the results of Section 3 in the theorem:

Theorem 5.3. Suppose $p(\rho)$ satisfies (H1), (H2) and (H3). If the system (1.1) is strictly hyperbolic or the initial data $\left(\rho_{ \pm}, u_{ \pm}\right)$satisfy $(5.18)$, then the boundary-value problem $(\mathcal{P})$ has a solution $(\rho, u)$ which is the limit of the solutions of the problems $\left(\mathcal{P}_{\varepsilon}\right)$ as $\varepsilon \rightarrow 0$. The function $(\rho, u)$ has the structure stated in Theorem 4.7 and does not contain a vacuum. $(\rho(x / t), u(x / t))$ is a solution of the Riemann problem (1.1), (1.2).

Acknowledgment. I would like to thank Professor A. E. Tzavaras, my thesis advisor. He introduced me to this problem and gave me valuable remarks on this work. Research was partially supported by the National Science Foundation under Grant DMS-9505342.

\section{REFERENCES}

[1] C. M. Dafermos, Solution of the Riemann problem for a class of hyperbolic conservation laws by the viscosity method, Arch. Rational Mech. Analysis 52, 1-9 (1973)

[2] C. M. Dafermos, Admissible wave fans in nonlinear hyperbolic systems, Arch. Rational Mech. Analysis 106, 243-260 (1989)

[3] A. S. Kalasnikov, Construction of generalized solutions of quasi-linear equations of first order without convexity conditions as limits of solutions of parabolic equations with a small parameter, Dokl. Akad. Nauk SSSR 127, 27-30 (1959)

[4] T. P. Liu, The Riemann problem for general $2 \times 2$ conservation laws, Trans. Amer. Math. Soc. 199, 89-112 (1974)

[5] T. P. Liu, Admissible solutions of hyperbolic conservation laws, Memoirs Amer. Math. Soc. 240, $1-78(1981)$

[6] P. H. Rabinowitz, Théorie de Degré Topologique et Applications à des Problèmes aux Limites non Linéaires, rédigé par H. Berestycki, Laboratoire d'Analyse Numérique, Université Paris VI, 1-300 (1975)

[7] M. Slemrod, Resolution of the spherical piston problem for compressible isentropic gas dynamics via a self-similar viscous limit, Proc. Roy. Soc. Edinburgh Sect. A 126, 1309-1340 (1996)

[8] M. Slemrod and A. E. Tzavaras, A limiting viscosity approach for the Riemann problem in isentropic gas dynamics, Indiana Univ. Math. J. 38, 1047-1074 (1989)

[9] A. E. Tzavaras, Elastic as limit of viscoelastic response in a context of self-similar viscous limit, J. Diff. Equations 123, 305-341 (1995)

[10] A. E. Tzavaras, Wave interactions and variation estimates for self-similar zero-viscosity limits in systems of conservation laws, Arch. Rational Mech. Anal. 135, 1-60 (1996)

[11] A. I. Volpert, The spaces $B V$ and quasilinear equations, Math. USSR Sbornik (N.S.) 73 (115), 255-302 (1967) 